\title{
LA MEDIAZIONE AL TEMPO DEL COVID-19 1
}

\section{MEDIATION IN TIMES OF COVID-19}

\section{Carlo Pilia}

Docente di diritto civile e tutele dei diritti nell'Università di Cagliari, Italia. E-mail: carlopiliaprof@gmail.com

RIASSUNTO: La pandemia Covid-19 sta avendo un impatto devastante sulle società e gli ordinamenti giuridici sia interni che internazionali. La normativa straordinaria ha dovuto considerare, tra gli altri aspetti, l'impossibilità e, comunque, l'estrema difficoltà di fare fronte ai pregressi impegni giuridici. Si è perciò resa necessaria l'introduzione di meccanismi di sospensione e differimento delle scadenze, oltre che di esenzione o almeno limitazione della responsabilità. Nello scenario emergenziale, pertanto, la questione dell'obbligatorietà della mediazione ai tempi del Covid è di grande interesse sistematico e forte impatto pratico.

PAROLE CHIAVE: Pandemia; normativa straordinaria; mediazzione.

\begin{abstract}
The Covid-19 pandemic has a devastating impact in societies and in internal and international law. The extraordinary rules should consider, among other aspects, the impossibility and also the extreme difficulties to cope with the previous legal commitments. It is necessary to introduce instruments of suspension and postpone of deadlines, as well as some exceptions or, at least, limitations of responsibilities. In this emergency scenario, mandatory mediation becomes an important issue in times of Covid-19 and is of great systematic interest and strong practical impact.
\end{abstract}

KEY WORDS: Pandemic; extraordinary rules; mediation.

\footnotetext{
${ }^{1}$ Artigo recebido em 10/12/2020, sob dispensa de revisão.
} 


\section{Le esigenze confliggenti di tutela durante la pandemia.}

La pandemia Covid-19 sta avendo un impatto devastante sulle società e gli ordinamenti giuridici sia interni che internazionali. Per contrastare la diffusione dei contagi e salvaguardare la salute pubblica e degli individui, infatti, le autorità hanno dovuto dichiarare lo stato di emergenza e imporre una serie di misure sanitarie restrittive della circolazione e del normale svolgimento delle attività relazionali ${ }^{2}$.

La fase emergenziale si caratterizza per l'approvazione di soluzioni straordinarie che, nei momenti più acuti di picco nei contagi, hanno imposto l'isolamento domestico delle persone, con la serrata della maggior parte delle attività produttive, sia in ambito pubblico che in quello privato, ad eccezione di quelle ritenute essenziali. La chiusura generalizzata delle attività (c.d. lockdown) e, comunque, l'applicazione delle altre misure sanitarie restrittive dettate a tutela del preminente valore della salute hanno costretto all'urgente introduzione di disposizioni emergenziali che garantissero la coesione economica e sociale del Paese.

La normativa straordinaria ha dovuto considerare, tra gli altri aspetti, l'impossibilità e, comunque, l'estrema difficoltà di fare fronte ai pregressi impegni giuridici. Si è perciò resa necessaria l'introduzione di meccanismi di sospensione e differimento delle scadenze, oltre che di esenzione o almeno limitazione della responsabilità. In tal senso, sono state introdotte disposizioni di carattere sia procedimentale, che sostanziale che hanno avuto un notevole impatto sulla gestione del sistema delle tutele dei diritti ${ }^{3}$.

Nello scenario emergenziale, pertanto, la questione dell'obbligatorietà della mediazione ai tempi del Covid è di grande interesse sistematico e forte impatto pratico ${ }^{4}$.

\footnotetext{
${ }^{2}$ In Italia lo stato di emergenza sanitaria per il Covid-19 è stato deliberato dal Consiglio dei Ministri nella riunione del 31 gennaio 2020 e poi prorogato nella riunione del 29 luglio 2020 fino al 15 ottobre 2020 e nella riunione del 7 ottobre 2020 fino al 31 gennaio 2021. Con riferimento alle misure straordinarie di contrasto alla pandemia approvate dal Governo, v. Coronavirus, le misure adottate dal Governo | www.governo.it

${ }_{3}$ Sull'emergenza pandemica nell'ordinamento giuridico italiano, AA. Vv., Emergenza Covid-19. Speciale Uniti per l'Italia, vol. 1, 2 e 3, in Giustizia civile.com, in http://giustiziacivile.com/.; AA. Vv., Fascicolo speciale 2020. Le fonti normative nella gestione dell'emergenza Covid-19, 2020, in https://www.osservatoriosullefonti.it/archivi/archivio-fascicoli/1544-fascicolo-speciale-2020/file.

${ }^{4}$ G. FINOCCHIARO, Tutte in mediazione obbligatoria le cause per gli inadempimenti COVID-19, in Quotidiano giuridico, martedi 7 luglio 2020, in https://www.quotidianogiuridico.it/documents/2020/07/07/tutte-inmediazione-obbligatoria-le-cause-per-gli-inadempimenti-covid-19; R. NARDONE, La nuova ipotesi di mediazione obbligatoria: le cause per inadempimento da COVID-19 e le procedure di sfratto, in Il processo civile, 9 Settembre 2020, in http://ilprocessocivile.it/articoli/focus/la-nuova-ipotesi-di-mediazioneobbligatoria-le-cause-inadempimento-da-covid-19-e-le; F. VALERINI, Nuova ipotesi di mediazione obbligatoria: le controversie contrattuali da rispetto delle misure COVID-19, in Diritto e giustizia. Il quotidiano di informazione giuridica, 26 Giugno 2020, in
} 
Nell'obbligatorietà, infatti, almeno due sono i prismi dell'analisi, distinti e concorrenti, che corrispondono ad altrettante fonti normative dettate per rispondere ad esigenze di tutela, apparentemente confliggenti, che si sono manifestate nel tempo. Benché sollecitate, quasi imposte, dalle situazioni di crisi strutturali ed emergenziali, le prescrizioni sull'obbligatorietà della mediazione hanno una comune carica riformatrice della giustizia e, più in generale, della società civile.

Anzitutto, occorre muovere dalla considerazione che nell'ordinamento italiano già esisteva una disciplina che conteneva delle ipotesi di obbligatorietà della mediazione, sia imposta dalla legge che ordinata dal giudice, in relazione a numerose controversie civili e commerciali $^{5}$. Per queste ipotesi, il problema pratico dell'emergenza pandemica riguarda la difficoltà, se non addirittura l'impossibilità durante il lockdown, di promuovere la mediazione prima o durante il processo giudiziale. Si sono incontrate, in pratica, le medesime restrizioni che hanno impedito o, quantomeno, rallentato lo svolgimento dell'attività giurisdizionale.

In secondo luogo, bisogna pure tenere in conto che proprio per fronteggiare l'enorme conflittualità originata dalla pandemia, non gestibile in sede giudiziale, si è reso necessario attivare le tutele extragiudiziali $\mathrm{e}$, in particolare, la mediazione ${ }^{6}$. A tal fine, la legislazione emergenziale ha permesso di superare alcune difficoltà organizzative che limitavano

http://www.dirittoegiustizia.it/news/23/0000099251/Nuova_ipotesi_di_mediazione_obbligatoria_le_controve rsie_contrattuali_da rispetto_delle misure_COVID_19.html; A. SPEDICATO, Mediazione obbligatoria nelle controversie causate dal covid-19. Sì, ma restano i dubbi sui casi concreti, in Consulenza, mercoledì, 1 luglio 2020, in https://www.consulenza.it/Contenuti/News/News/5409/mediazione-obbligatoria-nelle-controversieca.

${ }^{5}$ Sull'obbligatorietà della mediazione $e x$ art. 5, decreto legislativo 4 marzo 2010 n. 28 , tra i numerosissimi contributi, v. G. IMPAGNATIELLO, La mediazione civile in Italia, in judicium.it, p. 174 ss.; L. GAROFALO, ADR e diritto di accesso alla giustizia: il difficile raccordo tra modello europeo e modello italiano di mediaconciliazione obbligatoria in Studi sull'integrazione europea, 2014 n. 2, p. 247 ss.; G. SPINA, in La nuova mediazione. Regole e tecniche dopo le modifiche introdotte dal "Decreto del Fare" (d.l. 69/2013, conv., con mod., in l. 98/2013) a cura di G. Falco, G. Spina, Milano, 2014, p. 235 ss.; R. TISCINI, La mediazione civile e commerciale, Composizione della lite e processo nel d.lgs n. 23/2010 e nel D.M. nn. 180/2010 e 145/2011, Torino, 2011, p. 109 ss.; E. MINERVINI, La "storia infinita" della mediazione obbligatoria, in Contratti, 2013, p. 1153 ss.

${ }^{6}$ Sull'esperimento della mediazione e delle tutele extragiudiziali ai tempi del Covid-19, v. S. ORTIS, Le conciliazioni da remoto: un nuovo modello di dialogo conciliativo fra interventi normativi e prassi nell'emergenza sanitaria, in Lavoro, Diritti, Europa, 2020, 20 ottobre 2020, in https://www.lavorodirittieuropa.it/dottrina/processo-del-lavoro/552-le-conciliazioni-da-remoto-un-nuovomodello-di-dialogo-conciliativo-fra-interventi-normativi-e-prassi-nell-emergenza-sanitaria; S.A. CERRATO, F. DE SANTIS, L'arbitrato fra lockdown e fase due: una proposta di intervento normativo, in AA. Vv., Emergenza Covid-19. Speciale Uniti per l'Italia, vol. 3, cit., p. 13 ss. 
l'utilizzazione della mediazione e, infine, l'ha imposta come obbligatoria per le controversie contrattuali scaturenti dalla crisi pandemica. L'obbligatorietà della mediazione, pertanto, allenta la pressione del contenzioso sui tribunali, permettendo di trovare soluzioni consensuali più rapide.

In tal senso, l'obbligatorietà della mediazione costituisce una soluzione normativa che percorre le fasi che precedono, accompagnano e seguono l'emergenza pandemica, con la finalità di ovviare sia alle criticità della giurisdizione, che soffre cronicamente limiti strutturali, e sia per garantire la coesione economica e sociale della comunità, soprattutto nei momenti di più accentuata crisi. In entrambi i momenti, le prescrizioni dell'obbligatorietà della mediazione contribuiscono a innescare importanti processi di riforma del sistema in crisi delle tutele.

2. L'accesso alla mediazione prima del Covid-19.

La disciplina italiana sulla mediazione nelle controversie civili e commerciali ${ }^{7}$ adottata in attuazione della direttiva $2008 / 52 / \mathrm{CE}^{8}$ prevede una pluralità di modalità di accesso che salvaguardano il diritto fondamentale di rivolgersi all'autorità giudiziaria ${ }^{9}$. Più precisamente, la direttiva ammette che la mediazione, quale procedimento strutturato con il quale le parti tentano su base volontaria e con l'assistenza del mediatore di comporre

\footnotetext{
${ }^{7}$ Decreto legislativo 4 marzo 2010, n. 28 recante "Attuazione dell'articolo 60 della legge 18 giugno 2009, $n$. 69 , in materia di mediazione finalizzata alla conciliazione delle controversie civili e commerciali". Sulla disciplina italiana, tra gli altri, v. AA. VV., "La nuova mediazione civile e commerciale" a cura di T. Galletto, Editore Aracne, Roma, 2014; AA. Vv., La mediazione per la composizione delle controversie civili e commercialia cura di M. Bove, Padova, 2011; AA. VV., Mediazione e conciliazione nel nuovo processo civile. Commento organico al d.lgs. 4 marzo 2010, $n$. 28, in materia di mediazione finalizzata alla conciliazione nelle controversie civili e commerciali cura di B. Sassani, F. Santagata, Roma, 2010; AA.Vv., La nuova disciplina della mediazione nelle controversie civili e commerciali. Commentario al D.Lgs. 4 marzo 2010, n. 28, a cura di A. Bandini, N. Soldati, Milano, 2010; AA. Vv., in Quaderni di conciliazione a cura di C. Pilia, Cagliari, 2010-2020.

${ }^{8}$ Direttiva 2008/52/CE del Parlamento Europeo e del Consiglio del 21 maggio 2008 relativa a determinati aspetti della mediazione in materia civile e commerciale. Con riferimento allo statuto europeo della mediazione, v. AA. Vv., Mediación, Justicia y Unión Europea a cura di I.C. Iglesias Canle, Valencia, 2014; AA. Vv., Proceso civil y mediación. Su analisis en la ley 5/2012, de mediacion en asuntos civiles y mercantiles diretto da A. Bonet Navarro, Thomson Reuters Aranzadi, 2013; AA.Vv., Mediación en asuntos civiles y mercantiles. Comentarios a la Ley 5/2012 diretto da L. García Villaluenga, C. Rogel Vide, coordinato da C. Fernández Canales, Reus, Madrid, 2012; AA. Vv., ADR e mediazione a cura di C. Sticchi Damiani, Torino, 2012; AA. Vv., Mediazione e conciliazione nelle controversie civili e commerciali, Rimini, 2011, p. 11 ss.; AA. Vv., Manuale della mediazione civile e commerciale. Il contributo del Notariato alla luce del d.lgs. $n$. 28/2010 a cura di M.L. Cenni, E. Fabiani, M. Leo, Napoli, 2012; G. EsPlugues, G.L. IgLesias, G. PalaO, Civil and commercial Mediation in Europe, vol. I, National Mediation Rules and Procedures, Cambridge-AntwerpPortland, 2013.

${ }^{9}$ Art. 5, par. 1, Direttiva 2008/52/CE.
} 
consensualmente le controversie ${ }^{10}$, sia compatibile con varie modalità di accesso: per iniziativa delle parti, sollecitazione del giudice o prescrizione normativa ${ }^{11}$, secondo le scelte di politica del diritto rimesse alle legislazioni attuative degli Stati membri ${ }^{12}$.

Nell'attuare la direttiva europea, il legislatore italiano ha dovuto considerare le criticità sia del sistema giudiziario, colpito da disfunzioni croniche e da un enorme carico di arretrati, e sia del sistema extragiudiziale delle tutele, che non aveva ancora una disciplina organica della mediazione, né delle altre misure compositive consensuali. Perciò, la scelta politica è stata quella di adottare un modello fortemente costrittivo di mediazione ${ }^{13}$, nelle modalità di accesso $^{14}$ e di conduzione ${ }^{15}$, oltre che nelle conseguenze negative per la mancata partecipazione $^{16}$ e collaborazione ${ }^{17}$ delle parti nel procedimento finalizzato al raggiungimento dell'accordo.

Più precisamente, l'esperimento della mediazione è stato imposto normativamente come condizione legale di procedibilità dell'azione giudiziale nella maggior parte di controversie civili e commerciali ${ }^{18}$. In tali casi, l'esperimento della mediazione è obbligatorio prima dell'attivazione del giudizio (cd. mediazione obbligatoria).

Inoltre, in tutte le controversie civili e commerciali aventi per oggetto diritti disponibili è attribuito al giudice il potere di ordinare alle parti di accedere alla mediazione, a pena di ulteriore improcedibilità dell'azione giudiziale ${ }^{19}$. Nei giudizi pendenti, fino al grado d'appello, il giudice può imporre la mediazione quando, valutata la natura della causa, lo

\footnotetext{
${ }^{10}$ Art. 3, lett. a), direttiva 2008/52/CE.

${ }^{11}$ Art. 5 direttiva 2008/52/CE.

12 Sui sistemi di accesso alla mediazione previsti dalla direttiva 2008/52/CE, v. D. DALFINO, "Accesso alla giustizia" e "Mediazione", nel quadro normativo europeo. El marco regulatorio de la mediación en la Unión Europea, in Mediación, Justicia y Unión Europea, cit., p. 125 ss.; G. SPINA, in La nuova mediazione. Regole e tecniche dopo le modifiche introdotte dal "Decreto del Fare" (d.l. 69/2013, conv., con mod., in l. 98/2013) a cura di G. Falco, G. Spina, cit., p. 235 ss.; C.P. Dell'AnNA, E. GASBARRINI, M. LEO, I. PAGANELLI, in Manuale della mediazione civile e commerciale. Il contributo del notariato alla luce del d.lgs. $n$. 28/2010, cit., p. 212 ss.; C. BESSO, L'attuazione della direttiva europea n. 52 del 2008: uno sguardo comparativo, cit., p. 873 ss.

${ }^{13}$ Cfr. C. PILIA, Il modello italiano di mediazione: obbligatorietà e volontarietà, in AA. Vv., Quaderni di conciliazione a cura di C. Pilia, 11, Cagliari, 2019, p. 77 ss.

${ }^{14}$ Art. 5 d.lgs. n. 28/2010.

15 Art. 1, d.lgs. n. 28/2010.

${ }^{16}$ Art. 8 d.lgs. n. 28/2010.

17 Art. 13 d.lgs. n. 28/2010.

18 Art. 5, comma 1-bis, d.lgs. 28/2010, che si riferisce alle controversie in materia di "condominio, diritti reali, divisione, successioni ereditarie, patti di famiglia, locazione, comodato, affitto di aziende, risarcimento del danno derivante da responsabilità medica e sanitaria e da diffamazione con il mezzo della stampa o con altro mezzo di pubblicità, contratti assicurativi, bancari e finanziari”'.
}

${ }^{19}$ Art. 5, comma 2, d.lgs. n. 28/2010. 
stato dell'istruzione e il comportamento delle parti, risulti probabile il raggiungimento di un accordo compositivo (cd. mediazione delegata).

L'esperimento della mediazione, ancora, può trovare fondamento in una clausola inserita negli atti costitutivi o statuti degli enti e nei contratti ${ }^{20}$. In tal modo, si assume convenzionalmente l'obbligo di attivare la mediazione, magari scegliendo pure l'organismo incaricato di gestire il procedimento compositivo, prima di attivare l'azione giudiziale (cd. mediazione concordata).

Infine, fuori dalle predette ipotesi, la mediazione può essere sempre promossa volontariamente da ciascuna delle parti nelle controversie civili e commerciali vertenti su diritti disponibili ${ }^{21}$. Il procedimento di mediazione, pertanto, può essere promosso sia prima dell'esperimento dell'azione giudiziale, che pendente il giudizio (cd. mediazione facoltativa).

Dalle rilevazioni dei dati statistici, pubblicati semestralmente dal Ministero della Giustizia $^{22}$, risulta l'assoluta preminenza delle mediazioni obbligatorie, che per circa l'ottanta per cento sono imposte per legge e per il dieci per cento ordinate dal giudice, mentre meno del due per cento sono quelle derivanti dalle clausole di mediazione. Le mediazioni facoltative, infine, coprono circa il dieci per cento dei procedimenti. L'utilizzazione della mediazione, peraltro, non è sostenuta solamente dalla previsione della condizione di procedibilità legale, giudiziale e convenzionale dell'azione, ma anche dall'applicazione di altre misure sia costrittive che premiali. Tra le prime, vi sono le sanzioni processuali ed economiche a carico della parte che senza giustificato motivo non partecipi alla mediazione ${ }^{23}$ ovvero rifiuti di aderire alla proposta risolutiva scritta formulata dal mediatore ${ }^{24}$, allorquando il giudice adotti una sentenza di contenuto corrispondente ${ }^{25}$. Tra le seconde, possono

\footnotetext{
${ }^{20}$ Art. 5, comma 5, d.lgs. n. 28/2010.

${ }^{21}$ Art. 2, comma 1, d.lgs. n. 28/2010.

22 I raccolti dal Ministero della Giustizia, ai sensi dell'art. 11, Decreto Ministero della Giustizia 18 ottobre 2010 n. 180, Regolamento recante la determinazione dei criteri e delle modalità di iscrizione e tenuta del registro degli organismi di mediazione e dell'elenco dei formatori per la mediazione, nonché l'approvazione delle indennità spettanti agli organismi, ai sensi dell'articolo 16 del decreto legislativo 4 marzo 2010, n. 28, sono pubblicati ogni semestre e resi disponibili all'indirizzo web istituzionale: Presentazione standard di PowerPoint (giustizia.it)

${ }^{23}$ Art. 8, comma 4-bis, d.lgs. n. 28/2010.

${ }^{24}$ Art. 11, comma 2, d.lgs. n. 28/2010.

${ }^{25}$ Art. 13, commi 1 e 2, d.lgs. 28/2010.
} 
menzionarsi le agevolazioni fiscali riconosciute in mediazione ${ }^{26}$ e le esenzioni dai costi di mediazione per le parti non abbienti ${ }^{27}$.

Sostanzialmente, grazie all'applicazione della principale misura dell'obbligatorietà si è registrato il dato consolidato di oltre centocinquantamila mediazioni civili e commerciali annue. Senza l'obbligatorietà, in particolare, il sistema della mediazione non sarebbe riuscito a partire, né sarebbe a tutt'oggi capace di sostenersi solo con quelle volontarie. Il successo del modello obbligatorio italiano ha assunto una primaria rilevanza nello scenario europeo, tanto da essere studiato ${ }^{28} \mathrm{e}$, per certi versi, ripreso anche in altre realtà nazionali ${ }^{29}$.

Durante l'ultimo decennio, anche nell'esperienza italiana si sono diffuse altre procedure compositive extragiudiziali, alcune imposte obbligatoriamente quali condizioni di procedibilità dell'azione. Le principali fattispecie riguardano le controversie in materia di consumo $^{30}$, con riferimento alle ADR nei settori delle comunicazioni elettroniche ${ }^{31}$, dell'energia, del gas e del servizio idrico integrato ${ }^{32}$, oltre che le mediazioni di consumo ${ }^{33}$. Parimenti rilevante è l'imposizione dell'obbligatorietà della negoziazione assistita nelle controversie civili e commerciali avente ad oggetto le domande di pagamento, a qualsiasi titolo, di somme non eccedenti i cinquantamila euro e per il risarcimento danni da circolazione di veicoli e natanti ${ }^{34}$. Anche in questi casi, l'obbligatorietà delle procedure compositive consensuali non ha contribuito solo ad alleviare l'enorme carico del contenzioso

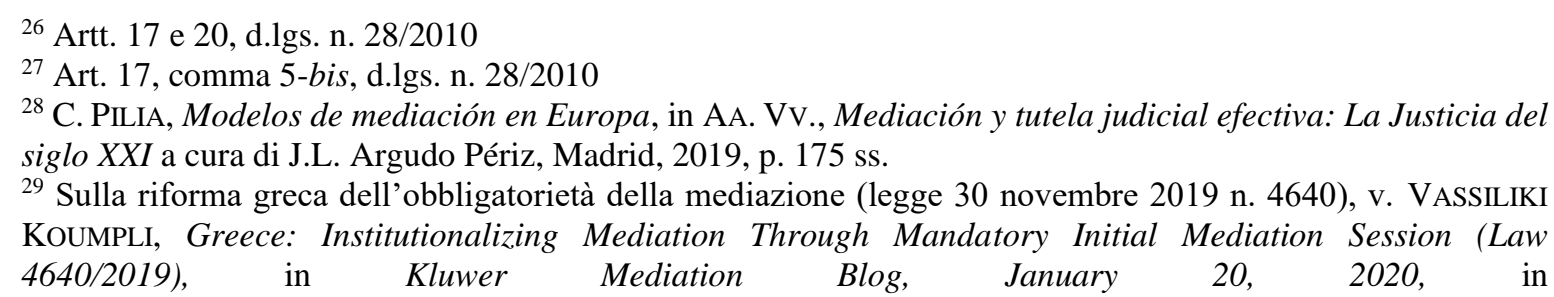
http://mediationblog.kluwerarbitration.com/2020/01/20/greece-institutionalizing-mediation-throughmandatory-initial-mediation-session-law-4640; MARINA PERRAKI, Mandatory mediation in Greece - the saga continuous, ivi, December 3, 2019.

${ }^{30}$ Art. 141, comma 6, cod. cons.

${ }^{31}$ Art. 1, comma 11, della legge 31 luglio 1997, n. 249, che prevede il tentativo obbligatorio di conciliazione nel settore delle comunicazioni elettroniche.

32 Art. 2, comma 24, lettera b), della legge 14 novembre 1995, n. 481, che prevede il tentativo obbligatorio di conciliazione nelle materie di competenza dell'Autorità per l'energia elettrica, il gas e il sistema idrico, e le cui modalità di svolgimento sono regolamentate dall'Autorità per l'energia elettrica, il gas e il sistema idrico con propri provvedimenti.

33 Art. 5, comma 1-bis, del decreto legislativo 4 marzo 2010, n. 28, che disciplina i casi di condizione di procedibilità con riferimento alla mediazione finalizzata alla conciliazione delle controversie civili e commerciali.

${ }^{34}$ Art. 3, decreto legge 12 settembre 2014, n. 132 recante "Misure urgenti di degiurisdizionalizzazione ed altri interventi per la definizione dell'arretrato in materia di processo civile", convertito con modificazioni dalla legge 10 novembre 2014, n. 162. 
giudiziario civile e commerciale, ma ha pure permesso di avviare subito l'infrastrutturazione del nuovo sistema delle tutele consensuali che si dovrebbe sviluppare pienamente anche su base volontaria.

3. L'introduzione di misure sospensive emergenziali.

Le prescrizioni sanitarie di contrasto ai contagi hanno provocato un generalizzato rallentamento e, nelle fasi acute di lockdown, persino una serrata delle attività lavorative, tanto in ambito pubblico quanto in quello privato. Di conseguenza. le autorità sono dovute intervenute mediante una serie di misure emergenziali di tipo giuridico, economico e sociale per limitare gli effetti negativi della crisi pandemica e dell'applicazione delle prescrizioni sanitarie.

Sul piano giuridico, l'approvazione di norme emergenziali è stata accompagnata dalla complessiva riorganizzazione in maniera agile dei processi produttivi (cd. smart working), mediante l'utilizzazione massiva di tecnologie e modalità di lavoro a distanza ${ }^{35}$. Sul piano normativo, anzitutto, è stata disposta la temporanea sospensione dei termini processuali e sostanziali per l'esercizio dei diritti ${ }^{36}$. Basti solo considerare che durante il lockdown anche

\footnotetext{
${ }^{35}$ Sullo smartworking durante la crisi pandemica, v. AA. VV., Covid-19 e diritti dei lavoratori, a cura di O. Bonardi, U. Carabelli, M. D’Onghia, L. Zoppoli, Roma, 2020; M. RUSSO, Emergenza lavoro agile nella P.A., in Emergenza Covid-19. Speciale Uniti per l'Italia, vol. 1, cit., p. 55 ss.; S. BINI, Lo smart working al tempo del coronavirus. Brevi osservazioni, in stato di emergenza, ivi, p. 67 ss.; S. CAIROLI, L'incentivo del lavoro agile nelle misure emergenziali di contrasto al COVID19: prime osservazioni, ivi, p. 87 ss.; I. SENATORI, Attivazione del lavoro agile e dei poteri datoriali nella decretazione emergenziale, ivi, p. 169 ss.; I. ALVINO, È configurabile un diritto del lavoratore al lavoro agile nell'emergenza Covid-19?, in Emergenza Covid-19. Speciale Uniti per l'Italia, vol. 2, cit., p. 101 ss.; C. MACCHIONE, Il lavoro agile ai tempi del Coronavirus, ivi, p. 107 ss.; M. FREDIANI, Il pubblico impiego in regime di coronavirus tra modifica d'imperio del luogo della prestazione e lavoro a domicilio, in Lavoro nella giurisprudenza, 5/2020, p. 511 ss.; C. DI CARLUCCIO, Emergenza epidemiologica e lavoro agile. Legislazione in materia di lavoro, in Rivista italiana di diritto del Lavoro, 1, 2020, p. 1 ss.

${ }^{36}$ Sulle disposizioni emergenziali in materia di processo civile e procedure ADR, v. E. DALMOTTO, I termini sostanziali e processuali civili nello sperabile crepuscolo del coronavirus. Tecniche della sospensione e altri rimedi, in Il caso.it, 4/5/20, in https://blog.ilcaso.it/news_937; A. PANZAROLA, M. FARINA, L'emergenza coronavirus ed il processo civile. Osservazioni a prima lettura, in Emergenza Covid-19. Speciale Uniti per l'Italia, vol. 1, cit., p. 3 ss.; F.M. GIORGI, Emergenza COVID-19 e prime osservazioni in ordine agli effetti sulle controversie di lavoro, ivi, p. 133 ss.; F. DANOVI, Procedimenti separativi su intesa e comparizione personale delle parti al tempo del Covid-19: la linea del Tribunale di Torino, in Famiglia e diritto, 5/2020, p. 431 ss.; G. BENEDETTI, La giustizia nel condominio in epoca di Covid-19, in Immobili \& proprietà, 6/2020, p. 391 ss.; A. CELESTE, Riunioni condominiali (inopportune) e sospensione dei termini (necessaria) per l'impugnazione delle delibere ... all'epoca del coronavirus, in Immobili \& proprietà, 6/2020, p. 364 ss.; M. TARANTINO, Il condominio ai tempi del coronavirus: assemblea in videoconferenza, in Condominioelocazione.it., 13 marzo 2020; G.P. MACAGNO, La legislazione d'emergenza e i processi fallimentari, in Fallimento, 5/2020, p. 697 ss.; F. DE SANTIS, La giustizia concorsuale ai tempi della pandemia, ivi, 2020, p. 612 ss.; R. BROGI, L'insolvenza all'epoca del Covid-19, ivi, p. 737 ss.
} 
gli uffici giudiziari, oltre che le amministrazioni pubbliche e gli studi professionali sono rimasti per mesi chiusi al pubblico.

La gran parte dei processi è stata rinviata nel tempo per l'impossibilità di celebrarli in maniera presenziale. Solo i casi urgenti e indifferibili sono stati trattati con modalità telematiche, rese possibili mediante la riorganizzazione agile dei servizi e l'utilizzazione delle tecnologie, anche per lo svolgimento delle udienze in videoconferenza ${ }^{37}$. Solo in minima misura e mediante l'utilizzazione di innovative modalità derogatorie rispetto a quelle presenziali ordinarie, quindi, si è riusciti a garantire una provvisoria tutela giudiziale dei diritti durante l'emergenza della crisi pandemica.

La medesima vicenda si è riproposta con riguardo alle tutele extragiudiziali e, in particolare, alla mediazione nelle controversie civili e commerciali. La legislazione emergenziale, infatti, come accaduto per $\mathrm{i}$ termini processuali $^{38}$, ha sospeso temporaneamente anche quelli della mediazione ${ }^{39}$. Successivamente sono state introdotte delle altre disposizioni emergenziali che hanno disciplinato alcuni aspetti della gestione a distanza del procedimento di mediazione ${ }^{40}$.

Più precisamente, l'iniziale disposizione emergenziale si riferisce alla mediazione obbligatoria e agli altri procedimenti compositivi imposti come condizione di procedibilità dell'azione giudiziale ${ }^{41}$. Il loro mancato esperimento, infatti, avrebbe finito per

\footnotetext{
${ }^{37}$ E. DALMOTTO, La sospensione straordinaria dei termini e il rinvio delle udienze nella prima e nella seconda fase del COVID-19, in Il caso.it, 24/06/20, in https://blog.ilcaso.it/news_970F; F. RICCIULLI, Il contraddittorio e il principio di oralità nel processo "emergenziale, nota a Consiglio di Stato 21 aprile 2020, n. 2539, sez. VI, in Il processocivile.it; A. STERLICCHIO DE CARLI, Giusto processo e oralità: il "contraddittorio cartolare coatto" nell'emergenza da COVID-19, nota a T.A.R. Napoli, 29 maggio 2020, n. 2074, sez. I, in Ilprocessotelematico.it; REDAZIONE SCIENTIFICA, Separazioni e divorzi: prosegue anche a Brescia la trattazione a distanza, nota al decreto 17 aprile 2020 del Presidente del Tribunale di Brescia, in Il familiatista.it.

${ }^{38}$ Art. 83 (Nuove misure urgenti per contrastare l'emergenza epidemiologica da COVID-19 e contenerne gli effetti in materia di giustizia civile, penale, tributaria e militare), Decreto Legge 17 marzo 2020, n. 18 Misure di potenziamento del Servizio sanitario nazionale e di sostegno economico per famiglie, lavoratori e imprese connesse all'emergenza epidemiologica da COVID-19, convertito con modificazioni dalla L. 24 aprile 2020, n. 27.

${ }^{39}$ Art. 83, comma 20, d.1. n. 18/2020.

${ }^{40}$ Art. 83 (Nuove misure urgenti per contrastare l'emergenza epidemiologica da COVID-19 e contenerne gli effetti in materia di giustizia civile, penale, tributaria e militare), Decreto Legge 17 marzo 2020, n. 18 Misure di potenziamento del Servizio sanitario nazionale e di sostegno economico per famiglie, lavoratori e imprese connesse all'emergenza epidemiologica da COVID-19, convertito con modificazioni dalla L. 24 aprile 2020, n. 27.

${ }^{41}$ Art. 83, comma 20, d.1. n. 18/2020, <<Per il periodo di cui al comma 1 (dal 9 marzo al 15 Aprile 2020) sono altresì sospesi i termini per lo svolgimento di qualunque attività nei procedimenti di mediazione ai sensi del decreto legislativo 4 marzo 2010, n. 28, nei procedimenti di negoziazione assistita ai sensi del decreto-legge 12 settembre 2014, n. 132, convertito, con modificazioni, dalla legge 10 novembre 2014, n. 162, nonché in tutti
} 
compromettere l'accesso alla stessa tutela giudiziale dei diritti. Al fine di garantire l'esercizio dei diritti, perciò, durante la prima fase acuta di emergenza pandemica, è stata temporaneamente sospesa la durata dei termini delle tutele giudiziali e di quelle presupposte extragiudiziali. Lo stretto collegamento con il giudizio, in sostanza, ha giustificato l'applicazione della sospensione temporanea dei termini, tanto processuali, quanto della mediazione e dei procedimenti compositivi obbligatori.

Le più rigorose misure sanitarie restrittive, quantomeno per il periodo di lockdown, imponevano l'isolamento domiciliare e, comunque, vietavano gli assembramenti di persone. Tanto impediva la gestione presenziale anche delle attività inerenti sia ai processi, che alla mediazione e agli altri procedimenti compositivi che coinvolgono più persone, giudice $\mathrm{o}$ mediatore, avvocati, parti, periti, consulenti, testimoni, personale di cancelleria e segreteria.

Tuttavia, bisogna evidenziare come la gestione presenziale del processo costituiva, quantomeno fino all'introduzione delle disposizioni emergenziali sulle udienze in videoconferenza o mediante scambio di memorie difensive, l'unica modalità contemplata dal codice di rito per lo svolgimento dei giudizi. Solo con le norme emergenziali, infatti, sono state introdotte per la prima volta e in via provvisoria le modalità di gestione a distanza delle udienze giudiziali, per i diversi ambiti di contenzioso sia pubblico, penale, amministrativo, contabile e tributario, che privato, civile, commerciale e familiare ${ }^{42}$.

Per la mediazione e gli altri meccanismi stragiudiziali di composizione dei conflitti, in realtà, la disciplina previgente già contemplava le modalità di gestione a distanza dei procedimenti, senza la necessità della riunione fisica delle persone. Questi meccanismi, infatti, si caratterizzano per la semplicità e l'informalità che sono realizzate anche attraverso gli strumenti di comunicazione a distanza impiegati per l'avvio, lo svolgimento e la definizione dei procedimenti compositivi. Malgrado la disciplina normativa ammettesse la gestione a distanza delle tutele extragiudiziali, nella pratica applicativa la mediazione e, tendenzialmente, le altre procedure stragiudiziali compositive continuavano a svolgersi prevalentemente in maniera presenziale. Si seguiva un approccio molto tradizionale e

\footnotetext{
$i$ procedimenti di risoluzione stragiudiziale delle controversie regolati dalle disposizioni vigenti, quando $i$ predetti procedimenti siano stati promossi entro il 9 marzo 2020 e quando costituiscono condizione di procedibilità della domanda giudiziale. Sono conseguentemente sospesi i termini di durata massima dei medesimi procedimenti.>>.

${ }^{42}$ Artt. 83 ss., d.l. n. 18/2020.
} 
conservatore in tutti i sistemi di gestione dei conflitti che, sostanzialmente, riproponevano l'impostazione presenziale coltivata dai giuristi nelle aule giudiziarie.

Del resto, quanto meno per la maggioranza dei giuristi, prima dell'emergenza pandemica era mancata l'effettiva frequenza di percorsi formativi teorici e pratici sulle piattaforme elettroniche per apprendere l'integrale gestione a distanza, anche con la videoconferenza, delle tutele giudiziali ed extragiudiziali. Si scontava, in definitiva, un'arretratezza e un'impreparazione culturale, professionale e tecnologica del sistema della giustizia nel suo insieme, anche dal punto di vista delle capacità di gestione agile del lavoro.

4. La mediazione a distanza durante il Covid-19.

La legislazione emergenziale, come accennato, ha espressamente previsto che anche $\mathrm{i}$ procedimenti di mediazione potessero essere gestiti a distanza, con l'accordo delle parti, nelle fasi del lockdown ${ }^{43}$. La gestione a distanza, infatti, non presupponendo la riunione nello stesso luogo del mediatore, degli avvocati e delle parti, non richiede spostamenti, né contatti fisici e, quindi, non importa tra i partecipanti alcun rischio di contagio del virus ${ }^{44}$.

L'utilizzazione delle tecniche di comunicazione a distanza, tuttavia, soddisfa plurime esigenze che non si esauriscono in quelle straordinarie insorte con la crisi pandemica, ma più in generale comprendono anche quelle ordinarie funzionali a semplificare, velocizzare $\mathrm{e}$ favorire la gestione agile della mediazione e dei procedimenti di tutela, sia extragiudiziali che giudiziali. Per queste ragioni funzionali, già la direttiva 2008/52/CE ha contemplato la possibilità di gestione a distanza della mediazione attraverso i mezzi elettronici ${ }^{45}$, che sono

\footnotetext{
43 Art. 83, d.1. n. 18/2020, comma 20-bis: "Nel periodo dal 9 marzo al 30 giugno 2020, gli incontri di mediazione in ogni caso possono svolgersi in via telematica con il preventivo consenso di tutte le parti coinvolte nel procedimento. Anche successivamente a tale periodo gli incontri potranno essere svolti, con il preventivo consenso di tutte le parti coinvolte nel procedimento, in via telematica, ai sensi dell'articolo 3 , comma 4, del decreto legislativo 4 marzo 2010, n. 28, mediante sistemi di videoconferenza. In caso di procedura telematica l'avvocato, che sottoscrive con firma digitale, può dichiarare autografa la sottoscrizione del proprio cliente collegato da remoto ed apposta in calce al verbale ed all'accordo di conciliazione. Il verbale relativo al procedimento di mediazione svoltosi in modalità telematica è sottoscritto dal mediatore e dagli avvocati delle parti con firma digitale ai fini dell'esecutività dell'accordo prevista dall'articolo 12 del decreto legislativo 4 marzo 2010, n. 28".

${ }^{44}$ E. DALmOTTO, L'arbitrato, la mediazione, la negoziazione assistita e gli altri ADR durante il COVID-19, in Ilcaso.it, 29/07/2020, in https://blog.ilcaso.it/news_980/29-0720/L\%E2\%80\%99arbitrato_la_mediazione_la_negoziazione_assistita_e_gli_altri_ADR_durante_il_COVI D-19.

45 Considerando n. 9, direttiva 2008/52/CE: "La presente direttiva non dovrebbe minimamente impedire l'utilizzazione di tecnologie moderne di comunicazione nei procedimenti di mediazione".
} 
utili per garantire la semplicità, rapidità, economicità e facilità di accesso e utilizzo della mediazione, soprattutto nelle controversie transfrontaliere ${ }^{46}$.

La disciplina attuativa italiana, peraltro, ha previsto espressamente la possibilità di gestione a distanza della mediazione ${ }^{47}$, con l'applicazione di alcune garanzie dirette a favorire l'accessibilità e la sicurezza del servizio compositivo. Sono gli organismi di mediazione, infatti, che fissano le regole di funzionamento mediante proprio regolamento ${ }^{48}$ che, in ogni caso, deve permettere di presentare le istanze anche offline $e^{49} \mathrm{e}$, inoltre, contenere anche specifiche regole per la gestione a distanza dei procedimenti ${ }^{50}$, nel rispetto della riservatezza dei dati trattati ${ }^{51}$.

Nella materia del consumo, il legislatore europeo ha approvato un pacchetto legislativo che, per un verso, con la direttiva 2013/11/UE ${ }^{52}$ disciplina il diritto dei consumatori di accedere ai sistemi di risoluzione extragiudiziale delle controversie nei confronti dei professionisti ${ }^{53}$, imponendo requisiti di qualità armonizzati sia per gli organismi e sia per le procedure $\mathrm{ADR}^{54}$. Tali requisiti, tra l'altro, impongono l'accessibilità sia online che offline

\footnotetext{
${ }^{46}$ Art. 2, direttiva 2008/52/CE.

${ }^{47}$ Art. 3, comma 4, d.lgs. n. 28/2010.

${ }^{48}$ Art. 3, comma 1, d.lgs. n. 28/2010.

${ }^{49}$ Art. 7, comma 4, d.m. n. 180/2010 ss.mm.

${ }^{50}$ Art. 3, comma 4, d.lgs. n. 28/2010.

${ }^{51}$ Artt. 3 e 9, d.lgs. 28/2010 e art. 7, comma 7, d.m. n. 180/2010.

52 Direttiva 2013/11/UE del Parlamento Europeo e del Consiglio del 21 maggio 2013 sulla risoluzione alternativa delle controversie dei consumatori, che modifica il regolamento (CE) n. 2006/2004 e la direttiva 2009/22/CE (direttiva sull'ADR per $i$ consumatori). Al riguardo, v. AA. Vv., The new regulatory framework for consumer dispute resolution a cura di P. Cortés, Oxford, 2016; AA.Vv., Soluciones alternativas a los conflictos de consumo. Perfiles hispano-italianos diretto da J.L. Tomillo Urbina, Granada, 2016; AA. VV., La resolución de conflictos de consumo. La adaptación del derecho español al marco europeo de resolución alternativa $(A D R)$ y en línea (ODR), Thomson Reuters Aranzadi, 2018; AA. VV., ADR. Una giustizia complementare a cura di F. Danovi, F. Ferraris, Milano 2018; AA. Vv., Mediación, arbitraje y juriscicción en el actual paradigma de justicia coordinato da S. Barona Vilar, Thomson Reuters-Civitas, 2016; AA. VV., Soluciones alternativas a los conflictos de consumo diretto da J.L. Tomillo Urbina, coordinato da I. ALVAREZ VEGA e G. Capilli, Comares, 2016; AA. VV., Estudios sobre Justicia online diretto da E. VÁZQUEZ DE CASTRO, coordinato da C. FERNÁNDEZ CANALES, Comares, 2013; AA. VV., Alternative Dispute Resolution in European Administrative Law a cura di D.C. Dragos, B.Neamtu, Springer Berlino-Heidelberg, 2014; AA. Vv., Fodertics II: hacia una justicia 2.0 coordinato da F. BUENO DE MATA, Ratio Legis, 2014; AA. VV., Le online dispute resolution $(O D R)$ a cura di E, Minervini, Napoli, 2016; AA.VV., Consumer ADR in Europe a cura di C. HoDGE, I. BENÖHR, N. CREUZFELDT-BANDA, Oxford, 2012; AA. VV., ODR e tentativo obbligatorio di mediazione, in Le Corti salernitane, n. 1, Napoli, 2015.

${ }^{53}$ Artt. 1 e 5, Direttiva 2013/11/UE.

${ }^{54}$ Capo II Accesso e requisiti applicabili agli organismi e alle procedure ADR, artt. 5-12, direttiva 2013/11/UE.
} 
degli organismi ${ }^{55}$ e delle procedure ${ }^{56}$, che devono essere gestibili anche a distanza ${ }^{57}$ senza la necessità della presenza fisica delle parti ${ }^{58}$.

Per altro verso, con il collegato regolamento (UE) $524 / 2013^{59}$ è stata istituita la piattaforma elettronica $\mathrm{ODR}^{60}$ che costituisce il punto unico di accesso ${ }^{61}$ per la proposizione delle procedure ADR relative al mercato digitale del consumo europeo. Sulla piattaforma ODR, infatti, è disponibile il modulo elettronico da compilare per la presentazione dell'istanza ${ }^{62}$ che viene comunicata alle parti ${ }^{63}$ e agli organismi ADR competenti ${ }^{64}$ che devono essere registrati sulla stessa piattaforma ${ }^{65}$. Le parti e l'organismo, inoltre, possono decidere di avvalersi della piattaforma ODR, che è gratuita e multilingue ${ }^{66}$, anche per la gestione della procedura ADR di consumo ${ }^{67}$.

Le disposizioni comunitarie sulle ADR in materia di consumo, comprese quelle sull'accessibilità a distanza, sono state recepite dal legislatore italiano ${ }^{68}$ mediante una

\footnotetext{
${ }^{55}$ Art. 5, direttiva 2013/11/UE.

${ }^{56}$ Art. 8, direttiva 2013/11/UE.

57 Art. 8, lett. a), direttiva 2013/11/UE.

${ }^{58}$ Art. 20, par. 2, lett. f), direttiva 2013/11/UE.

${ }^{59}$ Regolamento (UE) n. 524/2013 del Parlamento Europeo e del Consiglio del 21 maggio 2013 relativo alla risoluzione delle controversie online dei consumatori e che modifica il regolamento (CE) n. 2006/2004 e la direttiva 2009/22/CE (regolamento sull'ODR per $i$ consumatori). Sulla fonte regolamentare, v. AA. VV., La resolución de conflictos con consumidores de la mediación a las ODR coordinata da I. Barral Viñals, Editorial Reus, 2018; AA. VV., La resolución alternativa de litigios en materia de consumo diretto da M.J. Ariza Colmenarejo, M. J. Fernández-Fígares Morales, Tomson Reuters Aranzadi, 2018; AA. Vv., La protección del consumidor en dos espacios de integración: Europa y América, Una perspectiva de Derecho internacional, europeo y comparado diretto da F. Esteban de la Losa, P. Cortés, Tirant lo Blanch, 2015.

${ }^{60}$ Art. 5, regolamento (UE) n. 524/2013.

${ }^{61}$ Art. 5, par. 2, regolamento (UE) n. 524/2013.

${ }^{62}$ Art. 8, regolamento (UE) n. 524/2013.

${ }^{63}$ Art. 9, par. 2, regolamento (UE) n. 524/2013.

${ }^{64}$ Art. 9, par. 6, regolamento (UE) n. 524/2013.

${ }^{65}$ Art. 5, par. 6, regolamento (UE) n. 524/2013.

${ }^{66}$ Art. 5, par. 2, regolamento (UE) n. 524/2013.

${ }^{67}$ Art. 10, lett. d), regolamento (UE) n. 524/2013.

68 Decreto legislativo 6 agosto 2015, n. 130 "Attuazione della direttiva 2013/11/UE sulla risoluzione alternativa delle controversie dei consumatori, che modifica il regolamento (CE) n. 2006/2004 e la direttiva 2009/22/CE (direttiva sull'ADR per i consumatori)".
} 
novellazione del codice del consumo ${ }^{69}$. Come accennato, seppur di regola volontario ${ }^{70}$, l'esperimento della procedura ADR costituisce condizione di procedibilità dell'azione giudiziale $^{71}$ con riferimento alle controversie relative ai settori delle comunicazioni elettroniche ${ }^{72}$ e dell'energia, gas e servizio idrico integrato ${ }^{73}$, oltre che per le mediazioni obbligatorie di consumo ${ }^{74}$.

Con modalità di comunicazione a distanza, inoltre, si gestisce la procedura di negoziazione assistita tramite avvocati introdotta nell'ambito del processo di degiurisdizionalizzazione del contenzioso $^{75}$, di regola su base volontaria ${ }^{76}$, per le controversie civili, commerciali e delle crisi familiari ${ }^{77}$. In tali, casi, infatti, il negoziato è condotto dagli avvocati, che si scambiano gli atti della procedura a distanza ${ }^{78}$, anche nei casi in cui essa costituisce condizione di procedibilità dell'azione giudiziale ${ }^{79}$.

Rispetto a un siffatto quadro normativo oramai consolidato, pertanto, è intervenuta la legislazione emergenziale che, dopo la temporanea sospensione della mediazione e delle altre procedure compositive obbligatorie, ne ha espressamente ammesso l'esperimento a

\footnotetext{
${ }^{69}$ Artt. 141-141-decies, costituenti il «Titolo II-bis - Risoluzione extragiudiziale delle controversie», della parte $\mathrm{V}$ (Associazioni dei consumatori e accesso alla giustizia) del codice del consumo. Sulla disciplina codicistica italiana, v. AA. VV., Le online dispute resolution $(O D R)$ a cura di E. MINERVINI, Napoli, 2016; AA. VV., ODR e tentativo obbligatorio di mediazione, in Le Corti salernitane, n. 1, Napoli, 2015; C. PILIA, L'attuazione in Italia della direttiva 2013/11/UE sulle ADR per i consumatori, in Quaderni di conciliazione a cura di C. Pilia, n. 5, Cagliari, 2017, p. 153 ss.; A.M. MANCALEONI, La risoluzione extragiudiziale delle controversie dei consumatori dopo la direttiva 2013/11/UE, in Europa e Diritto Privato, n. 3, 2017, p. 1064 ss.; V. MIRRA, I sistemi di alternative dispute resolution trovano nuovo vigore: il recepimento della direttiva ADR $e$ l'introduzione del nuovo "arbitro per le controversie finanziarie", in Riv. dell'arbitrato, n. 4, 2016, p. 693 ss.; F. MANOLITA, Dalle ADR offline alle procedure di online dispute resolution, in https://www.judicium.it/wpcontent/uploads/saggi/646/M.\%20Francesca.pdf, 2015, p. 1 ss.

${ }^{70}$ Art. 141, comma 4, cod. consumo.

${ }^{71}$ Art. 141, comma 6, cod. consumo.

72 Art. 141, comma 6, lett. b), cod. consumo.

${ }^{73}$ Art. 141, comma 6, lett. c), cod. consumo.

${ }^{74}$ Art. 141, comma 6, lett. a), cod. consumo.

${ }^{75}$ Decreto legge 12 settembre 2014, n. 132 convertito con modificazioni dalla legge 10 novembre 2014, n. 162, recante: "Misure urgenti di degiurisdizionalizzazione ed altri interventi per la definizione dell'arretrato in materia di processo civile". Su tale disciplina, tra i tanti, v. F.P. LUISO, La negoziazione assistita, in Le nuove leggi civili commentate, n. 4/2015, p. 649 ss.; C. PUNZI, La c.d. « degiurisdizionalizzazione » della giustizia civile, in Punzi, Il processo civile - Le riforme del quinquennio 2010 - 2014, Torino, 2015, p. 1 ss.; G. FREZZA, "Degiurisdizionalizzazione", negoziazione assistita e trascrizione, in Le nuove leggi civili commentate, $\mathrm{n}$. $1 / 2015$, p. 18 ss.
}

${ }^{76}$ Art. 2, d.1. n. 132/2014.

${ }^{77}$ Art. 6, d.1. n. 132/2014.

${ }^{78}$ Art. 4, d.1. n. 132/2014.

${ }^{79}$ Art. 3, d.1. n. 132/2014. 
distanza durante la pandemia, con l'accordo delle parti ${ }^{80}$. In effetti, le disposizioni in vigore già ammettevano la gestione a distanza delle procedure compositive extragiudiziali, ancorché la prevalente prassi fosse incline alle modalità presenziali di gestione. La citata disposizione emergenziale, pertanto, si è limitata a esplicitare e, per certi versi, sollecitare l'applicazione di una disciplina che era già in vigore, ancorché fino ad allora fosse rimasta scarsamente sfruttata.

Sempre per favorirne la gestione a distanza, la legislazione emergenziale ha apportato alla disciplina vigente alcune significative novità normative e organizzative che hanno permesso di superare la necessità dei contatti fisici implicati negli incombenti procedurali iniziali e finali. Più precisamente, le disposizioni emergenziali hanno permesso che la parte possa dare a distanza l'incarico all'avvocato affinché l'assista in mediazione ${ }^{81} \mathrm{e}$, in caso di accordo conciliativo, che il mediatore, le parti e gli avvocati possano sottoscriverlo a $\operatorname{distanza}^{82}$.

La soluzione innovativa che ammette la certificazione a distanza delle sottoscrizioni, peraltro, non trova applicazione soltanto per la mediazione e le altre procedure compositive extragiudiziali, ma anche per le procedure giudiziali ${ }^{83}$. In tal senso, si è assistito a una decisa

\footnotetext{
${ }^{80}$ M. MARINARO, Semplificato l'iter per la mediazione, videoconferenza e accordo "a distanza», in Guida al diritto del 30 maggio 2020, p. 89 ss.

${ }^{81}$ Art. 83, comma 20-bis, D.L. 17/2020: "Nel periodo dal 9 marzo al 30 giugno 2020, gli incontri di mediazione in ogni caso possono svolgersi in via telematica con il preventivo consenso di tutte le parti coinvolte nel procedimento. Anche successivamente a tale periodo gli incontri potranno essere svolti, con il preventivo consenso di tutte le parti coinvolte nel procedimento, in via telematica, ai sensi dell'articolo 3, comma 4, del decreto legislativo 4 marzo 2010, $n$. 28, mediante sistemi di videoconferenza. In caso di procedura telematica l'avvocato, che sottoscrive con firma digitale, può dichiarare autografa la sottoscrizione del proprio cliente collegato da remoto ed apposta in calce al verbale ed all'accordo di conciliazione. Il verbale relativo al procedimento di mediazione svoltosi in modalità telematica è sottoscritto dal mediatore e dagli avvocati delle parti con firma digitale ai fini dell'esecutività dell'accordo prevista dall'articolo 12 del decreto legislativo 4 marzo 2010, n. 28".

${ }^{82}$ Art. 83, comma 20-ter, D.L. 18/2020: “Fino alla cessazione delle misure di distanziamento previste dalla legislazione emergenziale in materia di prevenzione del contagio da COVID-19, nei procedimenti civili la sottoscrizione della procura alle liti può essere apposta dalla parte anche su un documento analogico trasmesso al difensore, anche in copia informatica per immagine, unitamente a copia di un documento di identità in corso di validità, anche a mezzo di strumenti di comunicazione elettronica. In tal caso, l'avvocato certifica l'autografia mediante la sola apposizione della propria firma digitale sulla copia informatica della procura. La procura si considera apposta in calce, ai sensi dell'articolo 83 del codice di procedura civile, se è congiunta all'atto cui si riferisce mediante gli strumenti informatici individuati con decreto del Ministero della giustizia".

${ }^{83}$ Il problema della conciliazione giudiziale da remoto è stato risolto con la modifica dell'art. 88 , comma 2 , disp. att. c.p.c. introdotta con la 1. n. 70/2020 di conversione del d.1. n. 28/2020, nel quale è stato inserito un secondo comma così formulato: "Quando il verbale d'udienza, contenente gli accordi di cui al primo comma ovvero un verbale di conciliazione ai sensi degli articoli 185 e 420 del codice, è redatto con strumenti informatici, alla sottoscrizione delle parti, del cancelliere e dei difensori tiene luogo apposita dichiarazione
} 
innovazione e semplificazione procedimentale che, durante la crisi pandemica, ha permesso di ovviare alle restrizioni sanitarie nella circolazione delle persone e ai divieti di loro assembramenti. Sarebbe auspicabile che siffatte soluzioni procedimentali superassero la fase emergenziale, per divenire soluzioni strutturali, di "gestione agile delle tutele" tanto in ambito extragiudiziale, quanto giudiziale ${ }^{84}$. Tanto si rende necessario per affrontare l'ondata di conflittualità generata dalla crisi pandemica e, più in generale, per ammodernare e dare efficienza al sistema italiano delle tutele per tanti versi ancora troppo arretrato e farraginoso.

\section{Le disposizioni privatistiche.}

La legislazione emergenziale ha pure introdotto importanti disposizioni di carattere sostanziale, applicabili ai rapporti privatistici contrattuali e, più in generale, obbligatori che hanno subito gli effetti negativi della crisi pandemica ${ }^{85}$. Il rispetto delle prescrizioni sanitarie di contrasto ai contagi, nel limitare direttamente o indirettamente il normale svolgimento delle attività, ha complicato notevolmente e, in molti casi, reso impossibile l'esecuzione delle prestazioni dovute. L’inattuazione diffusa degli impegni giuridici ha ingenerato una

del giudice che tali soggetti, resi pienamente edotti del contenuto degli accordi, li hanno accettati. Il verbale di conciliazione recante tale dichiarazione ha valore di titolo esecutivo e gli stessi effetti della conciliazione sottoscritta in udienza". A.L. TERZI, I giudici del lavoro, l'udienza da remoto e la conciliazione giudiziale, in Questione giustizia, 7.7.2020, in https://www.questionegiustizia.it/articolo/i-giudici-del-lavoro-l-udienza-daremoto-e-la-conciliazione-giudiziale.

${ }^{84}$ E. DALMOTTO, La fine anticipata della seconda fase e la ripresa dei processi dopo il COVID-19, in Il caso.it, 27/06/20, in https://blog.ilcaso.it/news 971; M. MARINARO, La mediazione (telematica) dell'emergenza: un 'opportunità per la giustizia civile, in www.Judicium.it del $1^{\circ}$ giugno 2020, p. 1 ss.

${ }^{85}$ Sulle discipline emergenziali dei rapporti privatistici, tra i tantissimi contributi pubblicati, v. F. MACARIO, Per un diritto dei contratti più solidale in epoca di "coronavirus", in Emergenza Covid-19. Speciale Uniti per l'Italia, vol. 1, cit., p. 207 ss.; S. VERZONI, Gli effetti, sui contratti in corso, dell'emergenza sanitaria legata al COVID19, ivi, p. 213 ss.; A. DE MAURO, Pandemia e contratto: spunti di riflessione in tema di impossibilità sopravvenuta della prestazione, ivi, p. 227 ss.; V. CUFFARO, Le locazioni commerciali e gli effetti giuridici dell'epidemia, ivi, p. 233 ss.; F. GIGLIOTTI, Considerazioni in tema di impossibilità sopravvenuta, per emergenza epidemiologica, di prestazioni dello spettacolo e assimilate, ivi, p. 237 ss.; P. CHIARELLA, Solidarietà necessaria: erogazioni liberali nell'emergenza epidemiologica da Covid-19, ivi, p. 253 ss.; M. RubINo DE RITIS, Gli effetti della pandemia sull'economia digitale, ivi, p. 259 ss.; M. ATELL, Privato sociale alla prova della videoconferenza, ivi, p. 267 ss.; D. MAFFEIS, Problemi dei contratti nell'emergenza epidemiologica da Covid-19, in Emergenza Covid-19. Speciale Uniti per l'Italia, vol. 2, cit., p. 3 ss.; R.M. MAUGERI, L'emergenza Covid-19 e la sospensione dei mutui per l'acquisto della prima casa, ivi, p. 11 ss.; A.M. BENEDETTI, Il «rapporto» obbligatorio al tempo dell'isolamento: una causa (transitoria) di giustificazione?, ivi, p. 143 ss.; R. DI RAIMO, Le discontinuità che seguono i grandi traumi: pensando al credito (e al debito), mentre la notte è ancora fonda, ivi, p. 151 ss.; C. SCOGNAMIGLIO, L'emergenza Covid-19: quale ruolo per il civilista?, di Claudio Scognamiglio, ivi, p. 159 ss.; STUDIO BONELLI EREDE, Incidenza del coronavirus su alcune tipologie contrattuali, ivi, p. 169 ss.; R. SANTAGATA, Gli effetti del Coronavirus sui contratti turistici. Primi appunti, ivi, p. 225 ss.; U. SALANITRO, La gestione del rischio nella locazione commerciale al tempo del coronavirus, ivi, p. 235 ss.; M. ZACCHEO, Brevi riflessioni sulle sopravvenienze contrattuali alla luce della normativa sull'emergenza epidemiologica da Covid-19, ivi, p. 245 ss. 
generalizzata criticità, assai pericolosa sul piano della tenuta economica e sociale dell'ordinamento.

Con la più significativa disposizione privatistica di portata più generale, perciò, si è riconosciuto che dell'osservanza delle prescrizioni sanitarie di contrasto ai contagi si deve tenere conto nel valutare la responsabilità del debitore e le altre conseguenze dell'inadempimento ${ }^{86}$. In sostanza, a seconda dei casi, si introduce un'esimente ovvero un'attenuante di responsabilità per coloro che non hanno potuto adempiere in ragione delle prescrizioni sanitarie anticovid. Per l'ampia portata e la formulazione aperta, in effetti, la disposizione non si riferisce alla sola fattispecie codicistica dell'impossibilità sopravvenuta della prestazione, ma abbraccia un ventaglio molto più ampio di situazioni di graduata difficoltà. Inoltre, la disposizione rimane aperta anche sul piano rimediale. La risoluzione del contratto e l'estinzione dell'obbligazione, quali rimedi estintivi, non paiono soddisfare tutte le esigenze protettive, che richiedono anche rimedi correttivi e manutentivi del rapporto giuridico, da adeguare alle sopravvenienze pandemiche ${ }^{87}$.

A tal fine, la legislazione emergenziale comprende numerose altre disposizioni settoriali adottate con la specifica finalità di salvaguardare le relazioni giuridiche ${ }^{88}$. Così, tra gli altri, possono menzionarsi gli interventi privatistici in materia di rapporti di lavoro ${ }^{89}$,

\footnotetext{
${ }^{86}$ Art. 3, comma 6-bis, d.1. 23 febbraio 2020, n. 6 recante "Misure urgenti in materia di contenimento e gestione dell'emergenza epidemiologica da COVID-19", convertito in legge 5 marzo 2020, n. 13, dispone "6-bis. Il rispetto delle misure di contenimento di cui presente decreto è sempre valutata ai fini dell'esclusione, ai sensi e per gli effetti degli articoli 1218 e 1223 c.c., della responsabilità del debitore, anche relativamente all'applicazione di eventuali decadenze o penali connesse a ritardati o omessi adempimenti".

${ }^{87}$ Con riferimento a questa disposizione emergenziale, tra gli altri, v. L. GUERRINI, Coronavirus, legislazione emergenziale, e contratto: una fotografia, in Giustiziacivile.com, 2020, in www.giustiziacivile.com; F. VERZONI, Gli effetti, sui contratti in corso, dell'emergenza sanitaria legata al COVID-19, in Emergenza Covid19. Speciale Uniti per l'Italia, 1, cit., p. 213 ss.; M. ZACCHEO, Brevi riflessioni sulle sopravvenienze contrattuali alla luce della normativa sull'emergenza epidemiologica da Covid-19, in Emergenza Covid-19. Speciale Uniti per l'Italia, 2, cit., p. 245 ss.

${ }^{88}$ A. GENTILI, Una proposta sui contratti d'impresa al tempo del coronavirus, in AA. Vv., Emergenza Covid19. Speciale Uniti per l'Italia, vol. 3, cit., p. 383 ss.

${ }^{89}$ M. FATICATO, Ammortizzatori sociali per Covid-19 e la schizofrenia normativa: dalla conversione del d.l. Cura Italia al d.l. Rilancio, in AA. Vv., Emergenza Covid-19. Speciale Uniti per l'Italia, vol. 3, cit., p. 195 ss.; M. VerZARO, Il divieto di licenziamento nel decreto "Cura Italia”, ivi, p. 277 ss.; P. IERVOLINO, Sospensione (rectius nullità) dei licenziamenti economici per il COVID-19 e dubbi di legittimità costituzionale, ivi, p. 293
} 
bancari $^{90}$, di locazione ${ }^{91}$, dei servizi turistici ${ }^{92}$, etc. A tal fine, tuttavia, le autorità hanno dovuto adottare massicce politiche pubbliche di sostegno economico e assistenziale, a favore di ampie fasce di popolazione, lavoratori, professionisti e imprese. In tal modo, si sono intesi tutelare i fabbisogni primari delle persone in difficoltà, ma anche preservare il tessuto produttivo del Paese che è stato gravemente pregiudicato dal protrarsi della crisi pandemica $^{93}$.

Le misure economiche, per tanti versi, hanno inteso mitigare gli effetti pregiudizievoli della pandemia, nel tentativo di contenere l'accesa conflittualità che si prospetta sia nei rapporti privatistici, che verso la pubblica amministrazione, soprattutto quella tributaria.

6. L'obbligatorietà della mediazione per le controversie contrattuali originate dal Covid.

La grave crisi pandemica è causa del diffondersi di una profonda e generalizzata conflittualità che si insinua in tutti i settori, sia pubblici che privati, dell'ordinamento, minacciando la tenuta della coesione economica e sociale, sia interna che internazionale. Tendenzialmente, tutti i rapporti giuridici sono esposti agli effetti negativi della crisi pandemica e, pertanto, si rende necessario approntare meccanismi rapidi ed efficienti di tutela, anche avvalendosi delle soluzioni tecnologiche che si sono sperimentate nella fase emergenziale.

\footnotetext{
${ }^{90}$ L. SiCignano, La modifica unilaterale dei contratti bancari al tempo del Covid-19. Prime riflessioni, in AA. Vv., Emergenza Covid-19. Speciale Uniti per l'Italia, vol. 3, cit., p. 27 ss.; S. GUADAGNO, La conclusione dei contratti bancari all'epoca del covid-19: tra obblighi di forma e obblighi di comportamento, ivi, p. 53 ss.; R.M. MAUGERI, L'emergenza Covid-19 e la sospensione dei mutui per l'acquisto della prima casa, cit., p. 11 ss.

${ }^{91}$ L. MASSA, Oltre la Crisi. Lockdown e locazioni commerciali, in AA. Vv., Emergenza Covid-19. Speciale Uniti per l'Italia, vol. 3, cit., p. 309 ss.; F. FERRO LUZZI, Effetti secondari del Covid-19: la sopravvenuta, momentanea, modifica del dna dell'oggetto del contratto di locazione di esercizio commerciale, ivi, p. 315 ss.; U. SALANITRO, La gestione del rischio nella locazione commerciale al tempo del coronavirus, cit., p. 235 ss.; V. CUFFARO, Le locazioni commerciali e gli effetti giuridici dell'epidemia, cit., p. 233 ss.

92 R. Santagata, Gli effetti del Coronavirus sui contratti turistici. Primi appunti, cit., p. 225 ss.; V. VILlANOVA, I viaggi e gli spostamenti al tempo del Covid-19: qualche riflessione sullo scioglimento del contratto e sulla tutela di passeggeri e viaggiatori, in AA. Vv., Emergenza Covid-19 e questioni di diritto civile, a cura di E. Lucchini Guastalla, Torino, 2020, p. 71 ss.; A. PEPE, L'emergenza sanitaria da Coronavirus tra impossibilità sopravvenuta e impossibilità di utilizzazione della prestazione nei contratti di trasporto, di viaggio e del tempo libero (artt. 88 e 88 bis, d.l. 17 marzo 2020, n. 18 conv. con modif. dalla l. 24 aprile 2020, n. 27), in Nuove leggi civili commentate, 2020, p. 596 ss.

${ }^{93}$ G. ILIAS VigliotTI, Note sulla Cassa integrazione in deroga al tempo del diritto emergenziale, AA. Vv., Emergenza Covid-19. Speciale Uniti per l'Italia, vol. 3, cit., p. 251 ss.; M. FALSONE, Emergenza Covid e benefici dedicati al "settore privato": chi ne resta escluso?, in AA. Vv., Emergenza Covid-19. Speciale Uniti per l'Italia, vol. 2, cit., p. 95 ss.
} 
Si ipotizza, infatti, un'imponente ondata di contenziosi collegati alla crisi pandemica che ha alterato in maniera significativa sia la normalità della dinamica dei rapporti e sia l'equilibrio delle posizioni giuridiche che si avevano prima dell'affacciarsi del Covid. Non è pensabile che con il superamento della fase emergenziale si torni automaticamente, tantomeno in breve tempo, alla situazione precedente. Ne sono conferma le misure finanziarie straordinarie che a livello europeo, nazional e locale si stanno approvando per garantire la tenuta dell'ordinamento e la graduale ripresa delle attività, con la necessaria approvazione di riforme strutturali che interessano anche la giustizia e il sistema delle tutele giudiziali e stragiudiziali.

L'attuale sistema giurisdizionale, infatti, non appare in grado di reggere l'ondata di conflittualità scatenata dalla crisi pandemica. Per un verso, come accennato, le misure sanitarie anticovid hanno paralizzato e, comunque, rallentato enormemente l'ordinaria attività degli uffici giudiziari, così da provocare l'incremento degli arretrati e, per tanti versi, esasperare le croniche criticità strutturali della giurisdizione italiana. Tantomeno, gli uffici giudiziari sembrano in grado di fare fronte alla nuova conflittualità generata dalla pandemia, soprattutto se non si avviano le attese riforme di sistema che diano finalmente efficienza alla tutela giurisdizionale.

Anzitutto, bisogna intervenire sui meccanismi extragiudiziali di composizione dei conflitti che devono operare in via preventiva rispetto all'avvio del processo. Solo in tal modo, per un verso, si impedisce che si sovraccarichi e, quindi, si paralizzi l'attività giurisdizionale, impaludandola nella miriade di contenziosi che dovrebbero essere definiti con altri e più appropriati mezzi compositivi. Perlomeno, bisognerebbe provare a risolverli in via pregiudiziale. Per altro verso, in questo modo si eviterebbe che i conflitti si esasperino e finiscano per degenerare in una conflittualità diffusa che costituisce un'insidia temibile per la coesione economica e sociale del Paese.

Tra gli strumenti extragiudiziali, peraltro, sarebbero da favorire quelli finalizzati al raggiungimento di una soluzione amichevole di tipo consensuale e, in specie, la mediazione $^{94}$. I vantaggi dei meccanismi consensuali, infatti, consistono proprio nella capacità di rinsaldare le relazioni tra le parti in conflitto, accompagnandole verso nuovi

\footnotetext{
${ }^{94}$ Con riferimento agli interventi della legislazione emergenziale sulla mediazione, M. MARINARO, Obblighi contrattuali: la mediazione per risolvere le liti post-emergenza, in Guida al diritto del 25 luglio 2020, p. 79 ss.
} 
assetti reciprocamente vantaggiosi. Soprattutto in questa fase, infatti, la priorità del sistema delle tutele dovrebbe essere quella di tentare in via immediata di comporre amichevolmente i conflitti, con soluzioni che siano condivise dai contendenti.

In tal senso, nella legislazione emergenziale si ravvisa un'altra disposizione significativa che impone la condizione di procedibilità dell'esperimento della mediazione per le controversie contrattuali originate dall'applicazione delle prescrizioni sanitarie anticovid $^{95}$. Una nuova fattispecie di mediazione obbligatoria, quindi, che intende proprio deflazionare il contenzioso giudiziario, ma più in profondità sviluppare una dinamica amichevole di composizione dei conflitti in chiave solidaristica.

Una siffatta lettura teleologica della nuova obbligatorietà della mediazione per le controversie contrattuali Covid, peraltro, è suggerita dalla collocazione sistematica della disposizione emergenziale subito di seguito rispetto a quella sostanziale che ha introdotto l'esimente dalla responsabilità collegata all'osservanza delle prescrizioni sanitarie ${ }^{96}$. In partica, le due disposizioni si collegano funzionalmente nel responsabilizzare le stesse parti a trovare una soluzione amichevole per la crisi del rapporto, anche avvalendosi dell'assistenza del mediatore prima di rivolgersi all'autorità giudiziaria.

Si ravvisano le prime indicazioni che, sul piano sostanziale e procedimentale, tracciano le linee evolutive delle soluzioni amichevoli per la conflittualità pandemica, con il diretto e responsabile coinvolgimento delle parti contendenti. Esse, in primo luogo, devono farsi carico di trovare un componimento bonario, che permetta di superare la crisi mantenendo la relazione. Si tratta, com'è evidente, di disposizioni necessarie, ma ancora non sufficienti, in quanto occorrono riforme strutturali di portata generale improntate al principio solidaristico.

7. L'applicazione dei principi generali dell'ordinamento.

\footnotetext{
${ }^{95}$ L'art. 1, comma 1, della legge 25 giugno 2020, nel convertire con modifiche il d.1. 30 aprile 2020 n. 28 , ha disposto che "All'art.3 del d.l. 23.2 .20 n. 6 convertito con modificazioni dalla legge 5 marzo 2020 n.13 dopo il comma 6-bis è inserito il seguente: 6-ter nelle controversie in materia di obbligazioni contrattuali nelle quali il rispetto delle misure di contenimento di cui al presente decreto, o comunque disposte durante l'emergenza epidemiologica da COVID 19 sulla base di disposizioni successive, può essere valutato ai sensi dell'art.6 bis, il preventivo esperimento del procedimento di mediazione ai sensi del comma 1 bis dell'articolo 5 del Decreto Legislativo 28/10, costituisce condizione di procedibilità della domanda".

L'art. 91 del D.L. 17 marzo 2020, n. 18, ha introdotto il comma 6-bis, del citato art. 3, del D.L. 23 febbraio 2020 , n. 6, che dispone "6-bis. Il rispetto delle misure di contenimento di cui presente decreto è sempre valutata ai fini dell'esclusione, ai sensi e per gli effetti degli articoli 1218 e 1223 c.c., della responsabilità del debitore, anche relativamente all'applicazione di eventuali decadenze o penali connesse a ritardati o omessi adempimenti".

96 M. SCARSI, Mediazione e controversie covid 19, in Diritto.it, 8 luglio 2020, in https://www.diritto.it/mediazione-e-controversie-covid-19/.
} 
La crisi pandemica, per tanti versi, ha mostrato alcune criticità strutturali sul piano delle discipline sia sostanziali e sia delle tutele che segnano il nostro ordinamento. Probabilmente, l'emergenza Covid contribuisce al processo di riforma già in atto che, invero, da tempo si sarebbe dovuto avviare e che, comunque, non pare più differibile. All'attuazione delle riforme strutturali, a cominciare da quelle della giustizia, infatti, le istituzioni europee subordinano l'erogazione degli ingenti finanziamenti del recovery and Resilience fund per l'Italia ${ }^{97}$.

Evidente appare l'insufficienza delle disposizioni emergenziali rispetto agli ambiziosi obiettivi del Paese di comporre la conflittualità originata dalla pandemia e, in prospettiva, realizzare le attese riforme strutturali della giustizia. Tanto è dimostrato dalla necessità di fare ricorso, in via interpretativa, ai principi generali dell'ordinamento, cominciando da quelli di solidarietà e buona fede per gestire le sopravvenienze del Covid-19.

Da tempo sia la dottrina che la giurisprudenza avevano segnalato i limiti del sistema codicistico delle sopravvenienze contrattuali, tutto incentrato sulla risoluzione per impossibilità ed eccessiva onerosità sopravvenuta ${ }^{98}$. Da riconsiderare, da un lato, sono gli angusti spazi applicativi delle due tradizionali fattispecie tipizzate nel codice civile, da estendere a una serie di altre situazioni parimenti rilevanti, in quanto idonee ad alterare in maniera significativa l'iniziale equilibrio delle posizioni dei contraenti. Dall'altro, occorre pure ripensare l'apparato rimediale, ancora tutto incentrato sull' approccio demolitorio, che pone fine alla relazione giuridica alterata, piuttosto che intervenire per la sua conservazione mediante il ripristino dell'equilibrio delle posizioni delle parti coinvolte.

Prendendo spunto dai numerosi indici normativi contenuti sia nella disciplina codicistica dei contratti in generale e sia in quelle dei contratti speciali, ma soprattutto attraverso un'applicazione diretta dei principi generali di solidarietà e buona fede, autorevole dottrina ${ }^{99}$ ha sostenuto l'esigenza di un complessivo superamento del tradizionale approccio

\footnotetext{
${ }^{97}$ Con riferimento ai sistemi di finanziamento della crisi in Europa, v. AA. Vv., Andamento congiunturale, in Riv. dott. comm., 3, 2020, p. 525 ss.

${ }^{98}$ Sulle varie posizioni sostenute in dottrina e giurisprudenza, G. VETTORI, Persona e mercato al tempo della pandemia, in Persona e mercato, 2020, 1, p. 3 ss.; E. TUCCARI, Sopravvenienze e rimedi nei contratti di durata, Padova, 2018; R. SACCO, in R. Sacco, G. De Nova, Il contratto, Torino, 2016, p. 1708 ss.; F. GAMBINO, Problemi del rinegoziare, Milano, 2004; F. MACARIO, Rischio contrattuale e rapporti di durata nel nuovo diritto dei contratti: dalla presupposizione all'obbligo di rinegoziazione, in Riv. dir. civ., 2002, I, p. 63 ss; ID., Adeguamento e rinegoziazione nei contratti a lungo termine, Napoli, 1996.

${ }^{99}$ G. VetTORI, Persona e mercato al tempo della pandemia, cit., p. 5 ss.; F. MACARIO, Per un diritto dei contratti più solidale in epoca di “coronavirus”, cit., p. 207 ss.
} 
alle sopravvenienze contrattuali. In tal senso, i principi generali permettono sia di dare rilevanza a ulteriori fattispecie sopravvenute, diverse dall'impossibilità e dalla eccessiva onerosità sopravvenuta, che siano capaci di alterare in maniera significativa l'iniziale equilibrio contrattuale delle posizioni. Sempre i principi generali giustificano l'applicazione di rimedi nuovi di tipo conservativo-correttivo, finalizzati al ripristino dell'equilibrio delle posizioni. In tal senso, si afferma l'applicazione generale del rimedio della rinegoziazione attraverso cui le parti sono tenute a tentare una revisione consensuale del rapporto al fine di riportarlo all'iniziale posizione di equilibrio. Nell'esercizio di un siffatto rimedio finalizzato a realizzare una gestione solidale delle sopravvenienze, le parti si devono attenere al principio di buona fede. In mancanza, peraltro, spetta al giudice un intervento correttivo, diversamente atteggiato a seconda dei casi e degli interessi delle parti ${ }^{100}$.

Verso una tale riforma delle sopravvenienze, peraltro da tempo realizzata da altri ordinamenti nazionali sulla scorta di principi internazionali ${ }^{101}$, si stava avviando anche l'Italia con il progetto di legge governativa di revisione del codice civile ${ }^{102}$. L'emergenza pandemica, con ogni probabilità, darà una spinta decisiva per l'approvazione della riforma sostanziale delle sopravvenienze contrattuali nella disciplina codicistica ${ }^{103}$.

Non meno importante, tuttavia, è l'attesa riforma organica dei sistemi di tutela, a cominciare da quelli extragiudiziali di risoluzione consensuale, che si incentrano sul meccanismo della mediazione. Da anni si sollecita una riforma che ha formato oggetto di

\footnotetext{
${ }^{100}$ In proposito, v. L. GUERRINI, Coronavirus, legislazione emergenziale, e contratto: una fotografia, in AA. Vv., Emergenza Covid-19. Speciale Uniti per l'Italia, vol. 3, cit., p. p. 345 ss.; R. FRANCO, Emergenza. Diritto delle obbligazioni. Umanità, ivi, p. 359 ss.; A. GENTILI, Una proposta sui contratti d'impresa al tempo del coronavirus, ivi, p. 383 ss; A.M. BENEDETTI, Stato di emergenza, immunità del debitore e sospensione del contratto, ivi, p. 403 ss.; G. SPARANO, Effetti della pandemia sui contratti. Tesi dell'unitarietà, ivi, p. 413 ss.; R. MORELLO, Gli effetti sui contratti dell'emergenza sanitaria determinata dalla diffusione del coronavirus e l'applicazione dei rimedi previsti dal Codice Civile, ivi, p. 417 ss.

${ }^{101}$ F. MACARIO, Per un diritto dei contratti più solidale in epoca di "coronavirus, cit., p. 210.

${ }^{102}$ Sul disegno di legge governativo per la revisione del codice civile (DDL Senato n. 1151), presentato in Parlamento, v. F. AZZARI, Ricodificazione del diritto dei contratti: il ruolo della parte generale e l'influenza delle discipline di settore, in Contratto e impresa, 2019, n. 2, p. 571 ss.; L. BALESTRA, V. CUFFARO, C. SCognamiglio, G. Villa, Proposte di riforma del Codice civile: prime riflessioni, in Il Corriere Giuridico, n. 5/2019, p. 589 ss.; A. GENTILI, Il drafting legislativo (per una revisione del codice civile) - Introduzione; M. AINIS, Il buon legislatore e il codice civile; A. BELVEDERE, Alcuni aspetti del drafting legislativo; V. ROPPO, Note minime in tema di drafting legislativo e revisione del codice; H. SIMONETTI, Il drafting legislativo e la riforma del codice civile, contenuti negli Atti del Convegno "Il Drafting Legislativo (per una revisione del codice civile)" organizzato dalla Associazione dei Civilisti italiani a Roma il 25 gennaio 2019, resi disponibili online all'indirizzo: https://www.civilistiitaliani.eu/.

${ }^{103}$ Sul documento presentato dall'Associazione dei Civilisti italiani a sostegno della riforma codicistica delle sopravvenienze contrattuali, v. A.A. DOLMETTA, Il problema della rinegoziazione (ai tempi del coronavirus), in Giustiziacivile.com, 2020, p. 4 ss.
} 
elaborazione ad opera di autorevoli Commissioni di studio ${ }^{104}$. L'esigenza, infatti, è quella di dare finalmente effettività alle tutele ${ }^{105} \mathrm{e}$, quindi, garantire che le parti procedano esse stesse alla rinegoziazione del rapporto, non solo nei casi di sopravvenienze contrattuali, ma ogni qual volta insorga un conflitto, prima di attivare la tutela giudiziale. Al riguardo, le organiche discipline di matrice comunitaria della mediazione e dei sistemi ADR/ODR di consumo offrono un quadro di principi generali, regole di funzionamento e organizzazione dei servizi compositivi che possono utilmente essere impiegati per garantire l'effettività delle tutele dei diritti.

8. Verso una riforma strutturale delle tutele: tecnologie e meccanismi partecipativi.

L'imposizione dell'obbligo legale della mediazione, oltre che delle altre procedure compositive extragiudiziali, quali condizioni di procedibilità dell'azione giudiziale, in effetti, costituisce una misura costrittiva che, per tanti aspetti, si rende necessaria per sostenere l'indifferibile introduzione di un nuovo sistema di tutele consensuali che sia effettivamente praticato. L'obbligatorietà dell'esperimento della mediazione, come pure delle altre procedure compositive, peraltro, costituisce una misura funzionale non solo alla gestione dell'ondata di nuova conflittualità provocata dall'emergenza pandemica, ma più in generale al superamento dell'oramai cronica crisi strutturale del sistema italiano delle tutele, che si avvitano ancora sull'automatismo generalizzato dell'approdo giurisdizionale ${ }^{106}$.

L'obbligatorietà della mediazione, trascorsa oramai una decina di anni dalla sua introduzione in Italia, ha cominciato a dare i suoi frutti, permettendo di infrastrutturare il relativo sistema e avviare un nuovo approccio nella gestione consensuale dei conflitti. Il cambiamento è iniziato, ma i tempi di realizzazione sono lunghi e richiedono uno sforzo corale di tutti. Ancora una volta, la crisi pandemica può innescare una necessaria

\footnotetext{
104 In particolare, merita di essere segnalata la proposta di organica riforma degli strumenti di degiurisdizionalizzazione formulata dalla Commissione di studio presieduta dal prof. Guido Alpa, nominata dal Ministro della Giustizia con d.m. 7 marzo 2016, AA. Vv., Un progetto di riforma delle ADR, Napoli, 2017; G. AlPA, Commissione di studio per l'elaborazione di una organica disciplina volta alla «degiurisdizionalizzazione», in Rivista Trimestrale di Diritto e Procedura Civile, 2, 2017, p. 793 ss.

105 G. VetTori, Persona e mercato al tempo della pandemia, in Persona e Mercato, Saggi, 2020/1, p. 3 ss.

106 V. Maglione, Il lockdown toglie spazio alla mediazione. Ma intanto l'arretrato civile cresce, in Il Sole24ore, 23 novembre 2020, in https://www.ilsole24ore.com/art/il-lockdown-toglie-spazio-mediazione-maintanto-l-arretrato-civile-cresce-ADwazhl?refresh_ce=1.
} 
accelerazione dell'avviato processo di cambiamento, con una generalizzazione dell'utilizzo degli strumenti di autocomposizione negoziale dei conflitti sui diritti disponibili.

Invero, l'imposizione dell'obbligatorietà legale e di quella giudiziale costituisce il modello costrittivo resosi necessario in ragione dell'evidente carenza di cultura della mediazione e delle altre ADR/ODR, quali meccanismi complementari alla giurisdizione che concorrono a formare il sistema moderno della giustizia. Si potrebbe e, in prospettiva, si dovrà prescindere dall'imposizione eteronoma della mediazione se il suo esperimento diviene scelta volontaria e responsabile che trovi piena condivisione e valido sostegno da parte di tutta la società ${ }^{107}$.

L'approdo della riforma strutturale della giustizia, invero, dovrebbe essere l'assunzione generalizzata dell'impegno verso una gestione sostenibile e responsabile dei conflitti, come proiezione solidaristica del senso più profondo dell'appartenenza alla comunità. Le istituzioni, a cominciare da quelle preposte all'educazione e alla formazione, infatti, dovrebbero assumere un ruolo strategico in questo processo di cambiamento dalla mentalità del litigio, verso quella del dialogo e dell'accordo. Ma le stesse amministrazioni pubbliche nella gestione dei conflitti, a cominciare da quelli interni e con altre amministrazioni, dovrebbero seguire un approccio lealmente collaborativo. Lo stesso approccio si dovrebbe diffondere tra le imprese, anche attraverso meccanismi premiali di tipo reputazionale e competitivo da diffondere nelle dinamiche virtuose di mercato. I cittadini, infine, dovrebbero conoscere e sostenere il valore civico ed etico dell'approccio collaborativo, senza necessità che continui ad essere imposto dalla legge o da un ordine del giudice.

Solo in questa prospettiva di evoluzione collaborativa, per tanti aspetti utopica negli attuali scenari italiani di crisi strutturale e pandemica, l'introduzione generalizzata dell'obbligatorietà della mediazione per la gestione dei conflitti costituisce la premessa per il passaggio all'assunzione volontaria dell'impegno responsabile alla gestione collaborativa dei conflitti ${ }^{108}$. Almeno questo è l'auspicio di una stagione di riforme e miglioramenti in senso solidaristico per la giustizia e, più in profondità, per l'intera società.

\footnotetext{
${ }^{107}$ In proposito, v. AA. Vv., Mediación, conciliación y arbitraje tras la pandemia, diretto da G. Cerdeira Bravo de Mansilla, C. Pilia, Santiago, 2020.

${ }^{108}$ Sul nuovo approccio collaborativo nella gestione dei conflitti, v. E. LAUROBA, El derecho colaboratico como ADR y herramienta de la abogacía del futuro, in AA.Vv., Mediación, conciliación y arbitraje tras la pandemia, diretto da G. Cerdeira Bravo de Mansilla, C. Pilia, cit., p. 43 ss.
} 


\section{RIFERIMENTI}

Aa. Vv., "La nuova mediazione civile e commerciale” a cura di T. Galletto, Editore Aracne, Roma, 2014.

Aa. Vv., ADR e mediazione a cura di C. Sticchi Damiani, Torino, 2012.

Aa. Vv., ADR. Una giustizia complementare a cura di F. Danovi, F. Ferraris, Milano 2018.

Aa. Vv., Alternative Dispute Resolution in European Administrative Law a cura di D.C. Dragos, B.Neamtu, Springer Berlino-Heidelberg, 2014.

Aa. Vv., Andamento congiunturale, in Riv. dott. comm., 3, 2020.

Aa. Vv., Covid-19 e diritti dei lavoratori, a cura di O. Bonardi, U. Carabelli, M. D’Onghia, L. Zoppoli, Roma, 2020.

Aa. Vv., Emergenza Covid-19. Speciale Uniti per l'Italia, vol. 1,2 e 3, in http://giustiziacivile.com/.

Aa. Vv., Estudios sobre Justicia online diretto da E. Vázquez de Castro, coordinato da C. Fernández Canales, Comares, 2013.

Aa. Vv., Fascicolo speciale 2020. Le fonti normative nella gestione dell'emergenza Covid19, 2020, in https://www.osservatoriosullefonti.it/archivi/archivio-fascicoli/1544fascicolo-speciale-2020/file.

Aa. Vv., Fodertics II: hacia una justicia 2.0 coordinato da F. Bueno de Mata, Ratio Legis, 2014.

Aa. Vv., La mediazione per la composizione delle controversie civili e commercialia cura di M. Bove, Padova, 2011.

Aa. Vv., La protección del consumidor en dos espacios de integración: Europa y América, Una perspectiva de Derecho internacional, europeo y comparado diretto da F. Esteban de la Losa, P. Cortés, Tirant lo Blanch, 2015.

Aa. Vv., La resolución alternativa de litigios en materia de consumo diretto da M.J. Ariza Colmenarejo, M. J. Fernández-Fígares Morales, Tomson Reuters Aranzadi, 2018.

Aa. Vv., La resolución de conflictos con consumidores de la mediación a las ODR coordinata da I. Barral Viñals, Editorial Reus, 2018. 
Aa. Vv., La resolución de conflictos de consumo. La adaptación del derecho español al marco europeo de resolución alternativa $(A D R)$ y en línea $(O D R)$, Thomson Reuters Aranzadi, 2018.

Aa. Vv., Le online dispute resolution $(O D R)$ a cura di E, Minervini, Napoli, 2016.

Aa. Vv., Manuale della mediazione civile e commerciale. Il contributo del Notariato alla luce del d.lgs. n. 28/2010 a cura di M.L. Cenni, E. Fabiani, M. Leo, Napoli, 2012.

Aa. Vv., Mediación, arbitraje y juriscicción en el actual paradigma de justicia coordinato da S. Barona Vilar, Thomson Reuters-Civitas, 2016.

Aa. Vv., Mediación, conciliación y arbitraje tras la pandemia, diretto da G. Cerdeira Bravo de Mansilla, C. Pilia, Santiago, 2020.

Aa. Vv., Mediación, Justicia y Unión Europea a cura di I.C. Iglesias Canle, Valencia, 2014.

Aa. Vv., Mediazione e conciliazione nel nuovo processo civile. Commento organico al d.lgs. 4 marzo 2010, n. 28, in materia di mediazione finalizzata alla conciliazione nelle controversie civili e commerciali cura di B. Sassani, F. Santagata, Roma, 2010.

Aa. Vv., Mediazione e conciliazione nelle controversie civili e commerciali, Rimini, 2011, p. 11 ss.

Aa. Vv., ODR e tentativo obbligatorio di mediazione, in Le Corti salernitane, n. 1, Napoli, 2015.

Aa. Vv., Proceso civil y mediación. Su analisis en la ley 5/2012, de mediacion en asuntos civiles y mercantiles diretto da A. Bonet Navarro, Thomson Reuters Aranzadi, 2013.

Aa. Vv., Quaderni di conciliazione a cura di C. Pilia, Cagliari, 2010-2020.

Aa. Vv., Soluciones alternativas a los conflictos de consumo diretto da J.L. Tomillo Urbina, coordinato da I. Alvarez Vega e G. Capilli, Comares, 2016.

Aa. Vv., The new regulatory framework for consumer dispute resolution a cura di P. Cortés, Oxford, 2016.

Aa. Vv., Un progetto di riforma delle ADR, Napoli, 2017.

Aa.Vv., Consumer ADR in Europe a cura di C. Hodge, I. Benöhr, N. Creuzfeldt-Banda, Oxford, 2012.

Aa.Vv., La nuova disciplina della mediazione nelle controversie civili e commerciali. Commentario al D.Lgs. 4 marzo 2010, n. 28, a cura di A. Bandini, N. Soldati, Milano, 2010 . 
Aa.Vv., Mediación en asuntos civiles y mercantiles. Comentarios a la Ley 5/2012 diretto da

L. García Villaluenga, C. Rogel Vide, coordinato da C. Fernández Canales, Reus, Madrid, 2012.

Aa.Vv., Soluciones alternativas a los conflictos de consumo. Perfiles hispano-italianos diretto da J.L. Tomillo Urbina, Granada, 2016.

ALPA, G. Commissione di studio per l'elaborazione di una organica disciplina volta alla «degiurisdizionalizzazione», in Rivista Trimestrale di Diritto e Procedura Civile, 2, 2017.

ALVINO, I. È configurabile un diritto del lavoratore al lavoro agile nell'emergenza Covid19?, in Emergenza Covid-19. Speciale Uniti per l'Italia, vol. 2, in http://giustiziacivile.com/.

ATELLI, M. Privato sociale alla prova della videoconferenza, ivi.

AZZARI, F. Ricodificazione del diritto dei contratti: il ruolo della parte generale $e$ l'influenza delle discipline di settore, in Contratto e impresa, 2019, n. 2.

BALESTRA, L.; CUFFARO, V.; SCOGNAMIGLIO, C.; VILLA, G. Proposte di riforma del Codice civile: prime riflessioni, in Il Corriere Giuridico, n. 5/2019.

BENEDETTI, A. M. Il «rapporto» obbligatorio al tempo dell'isolamento: una causa (transitoria) di giustificazione?, ivi.

BENEDETTI, A. M., Stato di emergenza, immunità del debitore e sospensione del contratto, ivi.

BENEDETTI, G. La giustizia nel condominio in epoca di Covid-19, in Immobili \& proprietà, $6 / 2020$.

BESSO, C. L'attuazione della direttiva europea n. 52 del 2008: uno sguardo comparativo, cit.

BINI, S. Lo smart working al tempo del coronavirus. Brevi osservazioni, in stato di emergenza, ivi.

BROGI, R. L'insolvenza all'epoca del Covid-19, ivi.

CAIROLI, S. L'incentivo del lavoro agile nelle misure emergenziali di contrasto al COVID19: prime osservazioni, ivi. 
CELESTE, A. Riunioni condominiali (inopportune) e sospensione dei termini (necessaria) per l'impugnazione delle delibere ... all'epoca del coronavirus, in Immobili \& proprietà, $6 / 2020$.

CERRATO, S. A., DE SANTIS, F. L'arbitrato fra lockdown e fase due: una proposta di intervento normativo, in Aa. Vv., Emergenza Covid-19. Speciale Uniti per l'Italia, vol. 3 , in http://giustiziacivile.com/.

CHIARELLA, P. Solidarietà necessaria: erogazioni liberali nell'emergenza epidemiologica da Covid-19, ivi.

CUFFARO, V. Le locazioni commerciali e gli effetti giuridici dell'epidemia, cit.

DALFINO, D. “Accesso alla giustizia” e "Mediazione”, nel quadro normativo europeo. El marco regulatorio de la mediación en la Unión Europea, in Mediación, Justicia y Unión Europea a cura di I.C. Iglesias Canle, Valencia, 2014.

DALMOTTO, E. I termini sostanziali e processuali civili nello sperabile crepuscolo del coronavirus. Tecniche della sospensione e altri rimedi, in Il caso.it, 4/5/20, in https://blog.ilcaso.it/news_937.

DALMOTTO, E. L'arbitrato, la mediazione, la negoziazione assistita e gli altri ADR durante il COVID-19, in Ilcaso.it, 29/07/2020, in https://blog.ilcaso.it/news_980/29-0720/L\%E2\%80\%99arbitrato_la_mediazione_la_negoziazione_assistita_e_gli_altri_A DR_durante_il_COVID-19.

DALMOTTO, E. La fine anticipata della seconda fase e la ripresa dei processi dopo il COVID-19, in Il caso.it, 27/06/20, in https://blog.ilcaso.it/news_971.

DALMOTTO, E. La sospensione straordinaria dei termini e il rinvio delle udienze nella prima e nella seconda fase del COVID-19, in Il caso.it, 24/06/20, in https://blog.ilcaso.it/news_970F.

DANOVI, F. Procedimenti separativi su intesa e comparizione personale delle parti al tempo del Covid-19: la linea del Tribunale di Torino, in Famiglia e diritto, 5/2020.

DE MAURO, A. Pandemia e contratto: spunti di riflessione in tema di impossibilità sopravvenuta della prestazione, ivi.

DE SANTIS, F. La giustizia concorsuale ai tempi della pandemia, ivi, 2020. 
DELL'ANNA, C.P.; GASBARRINI, E.; LEO, M.; PAGANELLI, I. in Manuale della mediazione civile e commerciale. Il contributo del Notariato alla luce del d.lgs. $n$. 28/2010 a cura di M.L. Cenni, E. Fabiani, M. Leo, Napoli, 2012.

DI CARLUCCIO, C. Emergenza epidemiologica e lavoro agile. Legislazione in materia di lavoro, in Rivista italiana di diritto del Lavoro, 1, 2020.

DI RAIMO, R., Le discontinuità che seguono i grandi traumi: pensando al credito (e al debito), mentre la notte è ancora fonda, ivi.

DOLMETTA, A. A., Il problema della rinegoziazione (ai tempi del coronavirus), in Giustiziacivile.com, 2020.

ESPluGUES, G.; IGLESIAS, G.L.; PALAO, G. Civil and commercial Mediation in Europe, vol. I, National Mediation Rules and Procedures, Cambridge-AntwerpPortland, 2013.

FALSONE, M. Emergenza Covid e benefici dedicati al "settore privato": chi ne resta escluso?, in Aa. Vv., Emergenza Covid-19. Speciale Uniti per l'Italia, vol. 2, in http://giustiziacivile.com/.

FATICATO, M., Ammortizzatori sociali per Covid-19 e la schizofrenia normativa: dalla conversione del d.1. Cura Italia al d.1. Rilancio, in Aa. Vv., Emergenza Covid-19. Speciale Uniti per l'Italia, vol. 3, in http://giustiziacivile.com/.

FERRO LUZZI, F., Effetti secondari del Covid-19: la sopravvenuta, momentanea, modifica del dna dell'oggetto del contratto di locazione di esercizio commerciale, ivi.

FINOCCHIARO, G. Tutte in mediazione obbligatoria le cause per gli inadempimenti COVID-19, Quotidiano giuridico, martedì 7 luglio 2020, in https://www.quotidianogiuridico.it/documents/2020/07/07/tutte-in-mediazioneobbligatoria-le-cause-per-gli-inadempimenti-covid-19.

FRANCO, R., Emergenza. Diritto delle obbligazioni. Umanità, ivi.

FREDIANI, M. Il pubblico impiego in regime di coronavirus tra modifica d'imperio del luogo della prestazione e lavoro a domicilio, in Lavoro nella giurisprudenza, 5/2020. FREZZA, G., "Degiurisdizionalizzazione”, negoziazione assistita e trascrizione, in Le nuove leggi civili commentate, n. 1/2015. 
G. SPINA, in La nuova mediazione. Regole e tecniche dopo le modifiche introdotte dal "Decreto del Fare" (d.l. 69/2013, conv., con mod., in l. 98/2013) a cura di G. Falco, G. Spina, cit..

GAMBINO, F. Problemi del rinegoziare, Milano, 2004.

GAROFALO, L. ADR e diritto di accesso alla giustizia: il difficile raccordo tra modello europeo e modello italiano di media-conciliazione obbligatoria, Studi sull'integrazione europea, 2014 n. 2.

GENTILI, A. Una proposta sui contratti d'impresa al tempo del coronavirus, in Aa. Vv., Emergenza Covid-19. Speciale Uniti per l'Italia, vol. 3, in http://giustiziacivile.com/.

GENTILI, A., Il drafting legislativo (per una revisione del codice civile)-Introduzione; $\mathrm{M}$. Ainis, Il buon legislatore e il codice civile; A. Belvedere, Alcuni aspetti del drafting legislativo; V. Roppo, Note minime in tema di drafting legislativo e revisione del codice; H. Simonetti, Il drafting legislativo e la riforma del codice civile, contenuti negli Atti del Convegno "Il Drafting Legislativo (per una revisione del codice civile)" organizzato dalla Associazione dei Civilisti italiani a Roma il 25 gennaio 2019, resi disponibili online all'indirizzo: https://www.civilistiitaliani.eu/.

GENTILI, A., Una proposta sui contratti d'impresa al tempo del coronavirus, ivi.

GIGLIOTTI, F. Considerazioni in tema di impossibilità sopravvenuta, per emergenza epidemiologica, di prestazioni dello spettacolo e assimilate, ivi.

GIORGI, F.M. Emergenza COVID-19 e prime osservazioni in ordine agli effetti sulle controversie di lavoro, ivi.

GUADAGNO, S. La conclusione dei contratti bancari all'epoca del covid-19: tra obblighi di forma e obblighi di comportamento, ivi.

GUERRINI, L., Coronavirus, legislazione emergenziale, e contratto: una fotografia, in Aa. Vv., Emergenza Covid-19. Speciale Uniti per l'Italia, vol. 3, in http://giustiziacivile.com/.

IERVOLINO, P. Sospensione (rectius nullità) dei licenziamenti economici per il COVID-19 e dubbi di legittimità costituzionale, ivi.

ILIAS VIGLIOTTI, G. Note sulla Cassa integrazione in deroga al tempo del diritto emergenziale, in Aa. Vv., Emergenza Covid-19. Speciale Uniti per l'Italia, vol. 3, in http://giustiziacivile.com/. 
IMPAGNATIELLO, G. La mediazione civile in Italia, judicium.it, p. 174 ss.

ITALIA. Coronavirus, le misure adottate dal Governo. Governo Italiano: Presidenza del Consiglio dei Ministri. In: http://www.governo.it/it/coronavirus-misure-del-governo.

LAUROBA, E. El derecho colaboratico como ADR y herramienta de la abogacía del futuro, in Aa.Vv., Mediación, conciliación y arbitraje tras la pandemia, diretto da G. Cerdeira Bravo de Mansilla, C. Pilia, Santiago, 2020.

LUISO, F. P. La negoziazione assistita, in Le nuove leggi civili commentate, n. 4/2015.

MACAGNO, G.P. La legislazione d'emergenza e i processi fallimentari, in Fallimento, $5 / 2020$.

MACARIO, F. Rischio contrattuale e rapporti di durata nel nuovo diritto dei contratti: dalla presupposizione all'obbligo di rinegoziazione, in Riv. dir. civ., 2002, I; Id., Adeguamento e rinegoziazione nei contratti a lungo termine, Napoli, 1996.

MACARIO, F., Per un diritto dei contratti più solidale in epoca di "coronavirus", in Emergenza Covid-19. Speciale Uniti per l'Italia, vol. 1, in http://giustiziacivile.com/.

MACCHIONE, C., Il lavoro agile ai tempi del Coronavirus, ivi.

MAFFEIS, D. Problemi dei contratti nell'emergenza epidemiologica da Covid-19, in Emergenza Covid-19. Speciale Uniti per l'Italia, vol. 2, in http://giustiziacivile.com/.

MAGLIONE, V. Il lockdown toglie spazio alla mediazione. Ma intanto l'arretrato civile cresce, in Il Sole24ore, 23 novembre 2020, in https://www.ilsole24ore.com/art/illockdown-toglie-spazio-mediazione-ma-intanto-l-arretrato-civile-cresce-

ADwazh1?refresh_ce=1.

MANCALEONI, A. M. La risoluzione extragiudiziale delle controversie dei consumatori dopo la direttiva 2013/11/UE, in Europa e Diritto Privato, n. 3, 2017.

MANOLITA, F. Dalle ADR offline alle procedure di online dispute resolution, in https://www.judicium.it/wp-content/uploads/saggi/646/M.\%20Francesca.pdf, 2015.

MARINARO, M. La mediazione (telematica) dell'emergenza: un'opportunità per la giustizia civile, in www.Judicium.it del $1^{\circ}$ giugno 2020.

MARINARO, M. Obblighi contrattuali: la mediazione per risolvere le liti post-emergenza, in Guida al diritto del 25 luglio 2020.

MARINARO, M. Semplificato l'iter per la mediazione, videoconferenza e accordo «a distanza», in Guida al diritto del 30 maggio 2020. 
MASSA, L., Oltre la Crisi. Lockdown e locazioni commerciali, in Aa. Vv., Emergenza Covid-19. Speciale Uniti per l'Italia, vol. 3, in http://giustiziacivile.com/.

MAUGERI, R. M. L'emergenza Covid-19 e la sospensione dei mutui per l'acquisto della prima casa, cit.

MINERVINI, E. La “storia infinita” della mediazione obbligatoria, Contratti, 2013.

MIRRA, V. I sistemi di alternative dispute resolution trovano nuovo vigore: il recepimento della direttiva ADR e l'introduzione del nuovo "arbitro per le controversie finanziarie", in Riv. dell'arbitrato, n. 4, 2016.

MORELlO, R. Gli effetti sui contratti dell'emergenza sanitaria determinata dalla diffusione del coronavirus e l'applicazione dei rimedi previsti dal Codice Civile, ivi.

NARDONE, R. La nuova ipotesi di mediazione obbligatoria: le cause per inadempimento da COVID-19 e le procedure di sfratto, Il processo civile, 9 Settembre 2020, in http://ilprocessocivile.it/articoli/focus/la-nuova-ipotesi-di-mediazione-obbligatoriale-cause-inadempimento-da-covid-19-e-le.

ORTIS, S. Le conciliazioni da remoto: un nuovo modello di dialogo conciliativo fra interventi normativi e prassi nell'emergenza sanitaria, Lavoro, Diritti, Europa, 2020, 20 ottobre 2020, in https://www.lavorodirittieuropa.it/dottrina/processo-dellavoro/552-le-conciliazioni-da-remoto-un-nuovo-modello-di-dialogo-conciliativofra-interventi-normativi-e-prassi-nell-emergenza-sanitaria.

PANZAROLA, A.; FARINA, M. L'emergenza coronavirus ed il processo civile. Osservazioni a prima lettura, in Emergenza Covid-19. Speciale Uniti per l'Italia, vol. 1, in http://giustiziacivile.com/.

PEPE, A., L'emergenza sanitaria da Coronavirus tra impossibilità sopravvenuta e impossibilità di utilizzazione della prestazione nei contratti di trasporto, di viaggio e del tempo libero (artt. 88 e 88 bis, d.1. 17 marzo 2020, n. 18 conv. con modif. dalla 1. 24 aprile 2020, n. 27), in Nuove leggi civili commentate, 2020.

PERRAKI, Marina. Mandatory mediation in Greece - the saga continuous, ivi, December 3, 2019.

PILIA, C. Il modello italiano di mediazione: obbligatorietà e volontarietà, in Aa. Vv., Quaderni di conciliazione a cura di C. Pilia, 11, Cagliari, 2019. 
PILIA, C. L'attuazione in Italia della direttiva 2013/11/UE sulle ADR per i consumatori, in Quaderni di conciliazione a cura di C. Pilia, n. 5, Cagliari, 2017.

PILIA, C. Modelos de mediación en Europa, in Aa. Vv., Mediación y tutela judicial efectiva:

La Justicia del siglo XXI a cura di J.L. Argudo Périz, Madrid, 2019.

PUNZI C., La c.d. « degiurisdizionalizzazione » della giustizia civile, in Punzi, Il processo civile - Le riforme del quinquennio 2010 - 2014, Torino, 2015.

Redazione scientifica, Separazioni e divorzi: prosegue anche a Brescia la trattazione a distanza, nota al decreto 17 aprile 2020 del Presidente del Tribunale di Brescia, in $I l$ familiatista.it.

RICCIULLI, F. Il contraddittorio e il principio di oralità nel processo "emergenziale, nota a Consiglio di Stato 21 aprile 2020, n. 2539, sez. VI, in Il processocivile.it.

RUBINO DE RITIS, M. Gli effetti della pandemia sull'economia digitale, ivi.

RUSSO, M., Emergenza lavoro agile nella P.A., in Emergenza Covid-19. Speciale Uniti per l'Italia, vol. 1, in http://giustiziacivile.com/.

SACCO, R. in R. Sacco, G. De Nova, Il contratto, Torino, 2016.

SALANITRO, U. La gestione del rischio nella locazione commerciale al tempo del coronavirus, ivi.

SANTAGATA, R. Gli effetti del Coronavirus sui contratti turistici. Primi appunti, ivi.

SCARSI, M. Mediazione e controversie covid 19, in Diritto.it, 8 luglio 2020, in https://www.diritto.it/mediazione-e-controversie-covid-19/.

SCOGNAMIGLIO, C. L'emergenza Covid-19: quale ruolo per il civilista?, di Claudio Scognamiglio, ivi.

SENATORI, I. Attivazione del lavoro agile e dei poteri datoriali nella decretazione emergenziale, ivi.

SICIGNANO, L. La modifica unilaterale dei contratti bancari al tempo del Covid-19. Prime riflessioni, in Aa. Vv., Emergenza Covid-19. Speciale Uniti per l'Italia, vol. 3, in http://giustiziacivile.com/.

SPARANO, G. Effetti della pandemia sui contratti. Tesi dell'unitarietà, ivi.

SPEDICATO, A., Mediazione obbligatoria nelle controversie causate dal covid-19. Sì, ma restano i dubbi sui casi concreti, Consulenza, mercoledì, 1 luglio 2020, in 
https://www.consulenza.it/Contenuti/News/News/5409/mediazione-obbligatorianelle-controversie-ca.

SPINA, G. La nuova mediazione. Regole e tecniche dopo le modifiche introdotte dal "Decreto del Fare" (d.l. 69/2013, conv., con mod., in l. 98/2013) a cura di G. Falco, G. Spina, Milano, 2014.

STERLICCHIO DE CARLI, A. Giusto processo e oralità: il "contraddittorio cartolare coatto" nell'emergenza da COVID-19, nota a T.A.R. Napoli, 29 maggio 2020, n. 2074, sez. I, in Ilprocessotelematico.it.

STUDIO BONELLI EREDE, Incidenza del coronavirus su alcune tipologie contrattuali, ivi.

TARANTINO, M. Il condominio ai tempi del coronavirus: assemblea in videoconferenza, in Condominioelocazione.it., 13 marzo 2020.

TERZI, A. L. I giudici del lavoro, l'udienza da remoto e la conciliazione giudiziale, in Questione giustizia, 7.7.2020, in https://www.questionegiustizia.it/articolo/i-giudicidel-lavoro-l-udienza-da-remoto-e-la-conciliazione-giudiziale.

TISCINI, R. La mediazione civile e commerciale, Composizione della lite e processo nel d.lgs n. 23/2010 e nel D.M. nn. 180/2010 e 145/2011, Torino, 2011, p. 109 ss.

TUCCARI, E. Sopravvenienze e rimedi nei contratti di durata, Padova, 2018.

VALERINI, F. Nuova ipotesi di mediazione obbligatoria: le controversie contrattuali da rispetto delle misure COVID-19, Diritto e giustizia. Il quotidiano di informazione $\begin{array}{llll}\text { giuridica, } & 26 & \text { Giugno } & 2020,\end{array}$ http://www.dirittoegiustizia.it/news/23/0000099251/Nuova_ipotesi_di_mediazione obbligatoria_le_controversie_contrattuali_da_rispetto_delle_misure_COVID_19.htm 1 .

Vassiliki Koumpli, Greece: Institutionalizing Mediation Through Mandatory Initial Mediation Session (Law 4640/2019), in Kluwer Mediation Blog, January 20, 2020, in http://mediationblog.kluwerarbitration.com/2020/01/20/greece-institutionalizingmediation-through-mandatory-initial-mediation-session-law-4640.

VERZARO, M. Il divieto di licenziamento nel decreto “Cura Italia”, ivi, p. 277 ss.

VERZONI, F. Gli effetti, sui contratti in corso, dell'emergenza sanitaria legata al COVID19, in Emergenza Covid-19. Speciale Uniti per l'Italia, 1, in http://giustiziacivile.com/. 
VETTORI, G. Persona e mercato al tempo della pandemia, in Persona e Mercato, Saggi, 2020/1.

VILLANOVA V. I viaggi e gli spostamenti al tempo del Covid-19: qualche riflessione sullo scioglimento del contratto e sulla tutela di passeggeri e viaggiatori, in Aa. Vv., Emergenza Covid-19 e questioni di diritto civile, a cura di E. Lucchini Guastalla, Torino, 2020.

ZACCHEO, M., Brevi riflessioni sulle sopravvenienze contrattuali alla luce della normativa sull'emergenza epidemiologica da Covid-19, in Emergenza Covid-19. Speciale Uniti per l'Italia, 2, in http://giustiziacivile.com/. 